\title{
Bi-directional Beamformer Training for Dynamic TDD Networks
}

\author{
Praneeth Jayasinghe, Student Member, IEEE, Antti Tölli, Senior Member, IEEE, Jarkko Kaleva, Student Member, \\ IEEE and Matti Latva-aho, Senior Member, IEEE
}

\begin{abstract}
In dynamic time-division-duplexing (TDD) networks, the available resources per cell can be freely allocated to either uplink (UL) or downlink (DL) depending on the instantaneous traffic demand. Hence, complicated UL-DL and DL-UL interference scenarios arise due to simultaneous UL and DL data transmission in adjacent cells. In this paper, decentralized iterative beamformer designs are obtained for several traffic aware network optimization objectives such that only minimal information exchange is required among the coordinated base-stations (BSs) and user-equipments (UEs). Bi-directional forward-backward training via spatially precoded over-the-air pilot signaling is used to facilitate coordinated beamforming. This allows BSs and UEs to iteratively optimize their respective transmitters/receivers based on only locally measured reverse link pilot measurements. Novel bi-directional beamformer training strategies and methods for direct estimation (DE) of the stream specific beamformers are developed for each intermediate beamformer update in a limited and noisy pilot environment. The proposed signaling and DE schemes allow for non-orthogonal and overlapping pilots, which considerably reduces the resource coordination effort. Also, the decontamination ability of the proposed strategies are analyzed with limited pilot resources. The numerical examples illustrate the superior system performance of the proposed training and estimation framework in comparison to both the traditional stream-specific channel estimation method and an uncoordinated system.
\end{abstract}

Index Terms-dynamic or flexible TDD, coordinated beamforming, pilot decontamination, direct estimation, weighted queue minimization, weighted sum rate maximization.

\section{INTRODUCTION}

Mobile data traffic is expected to grow exponentially in the coming years due to ever-increasing smartphone usage, and massive demand for online video and cloud services [1], [2]. Future wireless mobile networks should be able to satisfy the increasing traffic demand which is asymmetric and bursty in nature. Consequently, small-cell deployment has been identified as a key research direction to fulfill these requirements. A large number of small cells with very short coverage area can be used in addition to the traditional macro/micro cells to deliver mobile data in high mobile data traffic areas [3], [4]. Furthermore, asymmetric mobile data traffic can be efficiently handled with time division duplexing

The authors are with Centre for Wireless Communications, University of Oulu P.O. Box 4500, FIN-90014 University of Oulu, Finland. Email: \{praneeth.laddu, antti.tolli, jarkko.kaleva, matti.latva-aho\}@oulu.fi

Parts of this work has been published in the IEEE ICC Workshop, London, U.K., 2015, the IEEE SPAWC, Stockholm, Sweden, 2015, the IEEE SPAWC, Sapporo, Japan, 2017, and the IEEE EuCNC, Oulu, Finland, 2017.

This research was supported by Finnish Funding Agency for Technology and Innovation (TEKES), Nokia Networks, Bittium, Keysight, Kyynel and MediaTek.
(TDD) by allocating resources in uplink (UL) and downlink (DL) directions based on the instantaneous traffic demand. Hence, TDD based small-cell networks provide several benefits, such as reduced complexity channel state information (CSI) estimation using channel reciprocity, flexible handling of dynamic traffic and easy frequency re-use planning [5], [6].

In a small cell network, imposing the same fixed UL/DL configuration over the network would greatly limit the overall resource utilization as the adjacent cells potentially have different instantaneous UL/DL traffic demand. Dynamic TDD can be used to overcome this problem by adjusting the UL/DL mode asynchronously based on the traffic state of each individual cell [6], [7]. Moreover, 3rd generation partnership project (3GPP) new radio (NR) standard has given special attention to fully dynamic or flexible TDD, which is an essential 5th generation mobile networks (5G) component [8], [9]. Consequently, there is a need for the new $5 \mathrm{G}$ air interfaces to meet the physical layer latency requirements without restrictions on UL/DL slot assignment [10].

Interference management in dynamic TDD systems becomes more challenging as complicated interference scenarios arise due to simultaneous UL and DL data transmission in adjacent cells. Specifically, the DL base station (BS) transmission may be interfering with the UL reception (BS-BS interference) and the UL user equipment (UE) transmission may be interfering with DL reception (UE-UE interference). Previous studies on dynamic TDD have focused on complicated time slot allocation algorithms that mitigate this cross-link interference [11]-[16]. In addition to increased spectral efficiency and improved reliability, spatial processing via multiple-input-multiple-output (MIMO) systems provides additional degrees of freedom to mitigate the detrimental interference both at the transmitter and receiver. Hence, the interference management in dynamic TDD based small-cell deployments is more efficient when both BSs and UEs are equipped with multiple antennas.

While the channel reciprocity can be utilized to acquire the CSI of the UE-BS and BS-BS links, a specific challenge of the dynamic TDD approach is to acquire the CSI between the mutually interfering UEs. Explicit feedback of the UEUE channels in addition to a full CSI exchange between BSs would be required to enable optimal beamformer design which renders the centralized design impractical. However, the coordinated beamformer design for dynamic TDD systems can be carried out in a decentralized manner with the help of bi-directional forward-backward (F-B) training via spatially precoded over-the-air (OTA) pilot signaling [17], [18]. In this 
case, it is essential to have fast beamformer convergence to implement these systems in practice with fading channels and to minimize the training overhead [17], [18].

Accurate CSI estimation is essential for any beamformer design. Hence, we need to employ distinguishable pilot sequences to estimate corresponding channels at the relevant nodes. However, the number of orthogonal pilot sequences that can be used for CSI estimation is constrained due to limited coherence time and coherence bandwidth of the wireless channel [19], [20]. Consequently, in dense networks, the number of orthogonal pilots is not sufficient to distinguish all possible channels. Hence, we must reuse pilots sequences or employ non-orthogonal pilots to estimate the CSI. This, on the other hand, causes the so-called pilot contamination effect, where the desired channel is polluted by other channels. Therefore, beamformer or CSI estimation should be robust against pilot contamination effect.

\section{A. Prior Work}

Most of the dynamic TDD studies are directed towards distributed dynamic time slot allocation algorithms to alleviate the cross-slot interference. For example, in [11]-[16] various time slot allocation strategies have been proposed and compared with the existing methods. In [21], small cell dynamic TDD transmissions have been investigated for heterogeneous networks by employing cell clustering and power control based interference cancellation scheme. In [22], an energy efficiency (EE) beamformer design was proposed with the cloud radio access network assumption, which requires centralized processing.

In the context of synchronous UL/DL ${ }^{1}$ TDD networks, numerous centralized and decentralized beamformer designs with different coordination assumptions have been considered to optimize network utilities such as weighted sum rate (WSR) maximization, EE, weighted queue minimization (WQM), and weighted sum mean square error (WSMSE) minimization, e.g., in [17], [23]-[31]. In [23], [24], the WSR maximization is carried out via WSMSE minimization and alternating optimization of the transmit precoders and receivers. Furthermore, the authors in [17] proposed a distributed CSI acquisition framework and novel fast converging strategies for iterative WSMSE based approach in a realistic multi-cell environment. Therein, bidirectional F-B training using spatially precoded pilots is employed to provide implicit exchange of intermediate beamformers between BSs and UEs, assuming that enough orthogonal pilots are available for stream specific precoded pilots. Further convergence improvements for WSR problem based on successive convex approximation (SCA) methods were proposed in [26], also with additional per user QoS/rate constraints. Recently, traffic aware transceiver design for weighted queue minimization has been investigated in [27]. Similarly to [17], [23], [24], [26], the decentralized solution in [27] was based on iterative evaluation of Karush-KuhnTucker (KKT) conditions of the optimization problem. The resulting beamformer structures were shown to be very similar

\footnotetext{
${ }^{1}$ The term, Synchronous UL/DL is used refer UL/DL transmission synchronously over the multicell network
}

to those corresponding to other optimization objectives such as WSR maximization, WSMSE minimization, etc. However, all the above studies assumed perfect channel estimation in their designs.

Practical implementation of coordinated precoding and CSI acquisition have been investigated in, e.g., [17], [32]-[35]. Moreover, the pilot contamination effect and possible ways to mitigate this impact have been studied, e.g., in [36]-[41]. An interesting approach to mitigate pilot contamination was proposed in [34], where direct least squares (LS) beamformer estimation from the contaminated UL/DL pilots was investigated. Moreover, there have been a few studies to mitigate pilot contamination by using pilot reuse algorithms with the use of channel statistics. For example, authors in [37] proposed a pilot reuse method utilizing spatial correlation properties. However, none of these studies consider dynamic TDD setting.

\section{B. Contributions}

Motivated by above concerns, we study several decentralized beamformer designs with different optimization objectives for multi-cell multi-user MIMO dynamic TDD systems. We consider WQM, and its special cases, queue weighted sum rate (QWSR) maximization and sum mean square error (SMSE) minimization objectives with power constraints at DL BSs and UL UEs. First, we improve the convergence properties of the WQM approach presented in [27] by reformulating it similarly to [26], and the problem is solved via iterative evaluation of KKT conditions leading to a distributed algorithm. Then, to facilitate practical implementation, we employ over-theair (OTA) signaling architecture as in [17], [18], [34]. More specifically, we employ precoded pilots to exchange the intermediate beamformers in both backward and forward direction iteratively. Hence, we consider a TDD frame to carry out both OTA bi-directional signaling and data transmission. Moreover, several bi-directional training iterations can be embedded into a TDD frame before the data transmission. A methodology was proposed in [18], to implement this TDD frame structure by adapting standardization works on 3GPP NR [9]. As the number of OTA signaling rounds is incresed, time remaining for the actual data transmission become shorter. Hence, the impact of OTA training overhead is investigated via numerical simulations. Then, we propose three different direct beamformer estimation methods to alleviate the pilot contamination effect. In these methods, we use the received precoded pilot information to estimate intermediate beamformers directly using LS based estimation without separately decorrelating individual pilot sequences. We refer these approaches as direct estimation (DE) methods. The DE approaches are compared with conventional stream-specific estimation (SSE) method, where the beamformers are constructed from the estimated equivalent (precoded) channel vectors. In both techniques, precoded pilot sequences are used to implicitly exchange information on beamformers and user-specific weights. However, when non-orthogonal pilots are used for the beamformer signaling, DE approach provides a better estimation due to the LS based estimation gain. Furthermore, the superiority of the DE method (versus SSE) is demonstrated analytically. 
Finally, to enhance the decontamination further, we consider a traditional pilot reuse approach for alleviating pilot overlap. A simple (but centralized) pilot reuse approach is proposed for complicated dynamic TDD setup based on large-scale fading information of the BS-UE and UE-UE channels.

Major contributions of this paper are summarized as follows:

- Fast converging iterative decentralized beamformer design is proposed for dynamic TDD system with the WQM objective.

- The proposed design is adapted to QWSR maximization and SMSE minimization objectives as special cases of WQM design.

- OTA bi-directional signaling architecture is proposed to implement beamformer designs in practice.

- Three DE-based beamformer estimation strategies are proposed to mitigate the pilot contamination.

- The superiority of DE over SSE is proven analytically.

- A centralized pilot reuse algorithm is proposed to dynamic TDD setting.

- Performance of the proposed methods are studied with numerical examples.

This article is an extended version of our previously published conference papers [42]-[45]. In [42], [43] we investigated WSR maximization problem for dynamic TDD network by employing bi-directional signaling to facilitate iterative decentralized design. Then, in [44], we studied the above system by employing non-orthogonal pilots. In [45] we investigated pilot decontamination methods using pilot reuse algorithms with the queue minimization objective.

\section{Organization and Notation}

The rest of the paper is organized as follows. Section II presents the system model that used for the rest of the article. In Section III, the proposed decentralized beamformer design is presented for dynamic TDD. Training and signaling architecture that supports decentralized beamformer design is addressed in Section IV. Pilot decontamination using DE is introduced in Section IV.A and three different DE strategies are presented in Sections IV.B-D. The DE comparison with the SSE approach is shown in Section IV.E. Pilot decontamination using pilot reuse methods are discussed in Sections IV.F. Complexity and overhead study is presented in Section IV.G. Finally, Section V presents the numerical examples and Section VI concludes the paper.

Notations: $\mathbb{C}^{m \times n}$ denotes an $m \times n$ matrix with elements in the complex field. Capital bold letters represent matrices, simple bold letters represent vectors and simple letters represent scalar variables. $(\cdot)^{-1},(\cdot)^{\mathrm{T}}$, and $(\cdot)^{\mathrm{H}}$ indicate inverse, transpose, and Hermitian of a matrix respectively. $\mathbb{E}\{$.$\} is the$ expectation of a random variable. Cardinality of a discrete set $\mathcal{A}$ is denoted as $|\mathcal{A}| \cdot \mathcal{C N}(x, y)$ denotes a complex Gaussian random variable with mean $x$ and variance $y$. A similar notation is valid when the variable is a vector or matrix.

\section{SySTEM MOdEL}

We consider a multi-cell multi-user MIMO system operating in dynamic TDD mode. The multi-cell network consists of

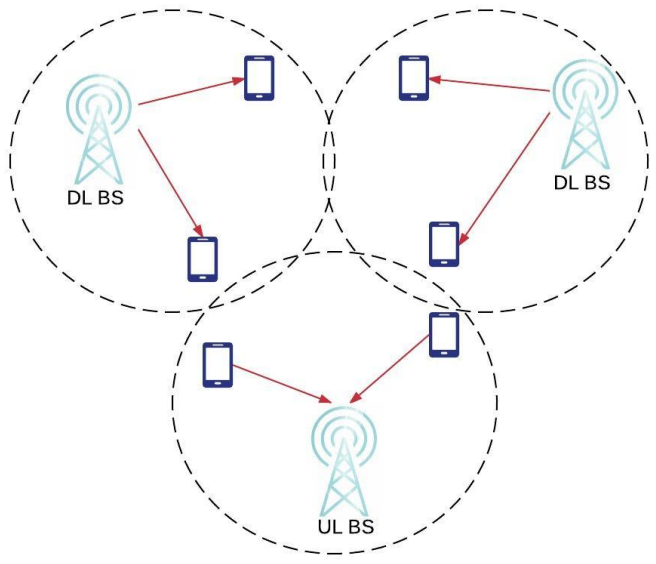

Fig. 1: Dynamic TDD system.

$N$ BSs and $K$ UEs. We denote the set of BS indices as $\mathcal{B}=\{1, \ldots, N\}$ while the set of UEs served by each BS $i$ is denoted by $\mathcal{U}_{i}$. The number of users served by each BS $i$ is denoted by $K_{i}=\left|\mathcal{U}_{i}\right|$. Also, the serving BS of the user $k$ is denoted as $i_{k}$. Each UE $k$ employs $N_{k}$ antenna elements, whereas each BS $i$ employs $M_{i}$ antenna elements. In a given time, a subset of base stations $\mathcal{B}_{U} \subseteq \mathcal{B}$ serves the uplink traffic and rest of the base stations $\mathcal{B}_{\mathrm{D}}=\mathcal{B} \backslash \mathcal{B}_{\mathrm{U}}$ are serving the DL traffic. The maximum number of spatial data streams allocated to UE $k \in \mathcal{U}_{i}$ is denoted by $L_{k} \leq \min \left(M_{i}, N_{k}\right)$. For notational simplicity, we define two sets $\mathcal{A}_{\mathrm{dl}}=\left\{j, l \mid j \in \mathcal{U}_{i}, l=1, \ldots, L_{j}, i \in \mathcal{B}_{\mathrm{D}}\right\}$ and $\mathcal{A}_{\mathrm{ul}}=\left\{j, l \mid j \in \mathcal{U}_{i}, l=1, \ldots, L_{j}, i \in \mathcal{B}_{\mathrm{U}}\right\}$. Here, $\mathcal{A}_{\mathrm{ul}}$ and $\mathcal{A}_{\mathrm{dl}}$ representing data stream indexing sets for the uplink and downlink transmissions, respectively. Furthermore, we assume the channels to be reciprocal in the UL and DL. The dynamic TDD model is illustrated in Fig. 1.

The received signal $\mathbf{x}_{k}^{(\mathrm{dl})} \in \mathbb{C}^{N_{k}}$ at the DL user $k$ can be expressed as

$$
\begin{aligned}
\mathbf{x}_{k}^{(\mathrm{dl})}= & \sum_{\{j, l\} \in \mathcal{A}_{\mathrm{dl}}} \mathbf{H}_{i_{j}, k}^{(\mathrm{dl})} \mathbf{m}_{j, l}^{(\mathrm{dl})} d_{j, l}^{(\mathrm{dl})} \\
& +\sum_{\{j, l\} \in \mathcal{A}_{\mathrm{ul}}} \mathbf{H}_{j, k}^{(\mathrm{ul}-\mathrm{dl})} \mathbf{m}_{j, l}^{(\mathrm{ul})} d_{j, l}^{(\mathrm{ul})}+\mathbf{z}_{k}^{(\mathrm{dl})},
\end{aligned}
$$

where $\mathbf{H}_{i_{j}, k}^{(\mathrm{dl})} \in \mathbb{C}^{N_{k} \times M_{i_{j}}}$ is the channel matrix between the DL BS $i_{j}$ and the UE $k, \mathbf{H}_{j, k}^{\text {(ul-dl) }} \in \mathbb{C}^{N_{k} \times N_{j}}$ is the interference channel matrix between the UL UE $j$ and the DL UE $k$. The transmit precoder for $l^{\text {th }}$ spatial data stream of the DL UE $j \in \mathcal{U}_{i}$ is denoted as $\mathbf{m}_{j, l}^{(\mathrm{dl})} \in \mathbb{C}^{M_{i}}$ and the transmit precoder for $l^{\text {th }}$ spatial data stream of the UL UE $j \in \mathcal{U}_{i}$ is $\mathbf{m}_{j, l}^{(\text {ul })} \in$ $\mathbb{C}^{N_{j}}$. Transmitted data symbols to DL UE $j$ in $l^{\text {th }}$ spatial stream and transmitted data symbols from UL UE $j$ in $l^{t h}$ spatial stream are denoted as $d_{j, l}^{(\mathrm{dl})}$ and $d_{j, l}^{(\mathrm{ul})}$, respectively. Here, the transmit data symbols are assumed to be independent and identically distributed with $\mathbb{E}\left\{\left|d_{j, l}^{(\mathrm{dl})}\right|^{2}\right\}=1$ and $\mathbb{E}\left\{\left|d_{j, l}^{(\mathrm{ul})}\right|^{2}\right\}=$ 1. We assume complex white Gaussian noise vector $\mathbf{z}_{k}^{(\mathrm{dl})} \in$ $\mathbb{C}^{N_{k}}$ with variance $\mathcal{N}_{0}$ per element. Similarly, the received 
signal $\mathbf{x}_{i}^{(\mathrm{ul})} \in \mathbb{C}^{M_{i}}$ at the uplink BS $i$ is given by

$$
\begin{aligned}
\mathbf{x}_{i}^{(\mathrm{ul})}= & \sum_{\{j, l\} \in \mathcal{A}_{\mathrm{ul}}} \mathbf{H}_{i, j}^{(\mathrm{ul})} \mathbf{m}_{j, l}^{(\mathrm{ul})} d_{j, l}^{(\mathrm{ul})} \\
& +\sum_{\{j, l\} \in \mathcal{A}_{\mathrm{dl}}} \mathbf{H}_{i_{j}, i}^{(\mathrm{dl}-\mathrm{ul})} \mathbf{m}_{j, l}^{(\mathrm{dl})} d_{j, l}^{(\mathrm{dl})}+\mathbf{z}_{i}^{(\mathrm{ul})},
\end{aligned}
$$

where $\mathbf{H}_{i, j}^{(\mathrm{ul})} \in \mathbb{C}^{M_{i} \times N_{j}}$ is the channel matrix between user $j$ and UL BS $i, \mathbf{H}_{i_{j}, i}^{(\mathrm{dl} \text {-ul })} \in \mathbb{C}^{M_{i} \times M_{i_{j}}}$ is the interference channel matrix between the DL BS $i_{j}$ serving user $j$ and the UL BS $i$. Also, we consider $\mathbf{z}_{i}^{(\mathrm{ul})} \in \mathbb{C}^{M_{i}}$ to be complex white Gaussian noise vector with variance $\mathcal{N}_{0}$ per element. Note that, due to the channel reciprocity, $\mathbf{H}_{i, j}^{(\mathrm{ul})}=\mathbf{H}_{i, j}^{(\mathrm{dll})^{\mathrm{T}}}$.

To estimate the received data through $l^{t h}$ spatial stream, DL UE $k$ employs a linear receiver $\mathbf{u}_{k, l}^{(\mathrm{dl})} \in \mathbb{C}^{N_{k}}$. Similarly, the UL BS $i_{k}$ employs a linear receiver $\mathbf{u}_{k, l}^{(\text {ul }} \in \mathbb{C}^{M_{i_{k}}}$ to decode the received data from UL user $k$ through the spatial stream l. Then, the estimated data at a $\mathrm{RX}^{2}$ node, corresponding to UL/DL data transmission can be written in a common form as $\hat{d}_{k, l}^{(a)}$. Here, for DL UE $k$, estimated data is given as $\hat{d}_{k, l}^{(\mathrm{dl})}=\mathbf{u}_{k, l}^{(\mathrm{dl})^{\mathrm{H}}} \mathbf{x}_{k}^{(\mathrm{dl})}$ whereas for UL BS $i_{k}$, the estimated data transmitted by UE $k$ is given as $\hat{d}_{k, l}^{(\mathrm{ul})}=\mathbf{u}_{k, l}^{(\mathrm{ul})^{\mathrm{H}}} \mathbf{x}_{i_{k}}^{(\mathrm{ul})}$. From here on, for simplicity, a generic supercript ' $a$ ' is introduced to represent both 'DL' and 'UL' directions.

The mean squared error (MSE) for $l^{\text {th }}$ stream of for UE $k$ corresponding to UL/DL data detection is defined as ${ }^{3}$

$$
\begin{aligned}
\epsilon_{k, l}^{(a)} & =\mathbb{E}_{d}\left[\left|d_{k, l}^{(a)}-\hat{d}_{k, l}^{(a)}\right|^{2}\right] \\
& =1-2 \Re\left(\mathbf{u}_{k, l}^{(a)}{ }^{\mathrm{H}} \mathbf{H}_{i_{k}, k}^{(a)} \mathbf{m}_{k, l}^{(a)}\right)+\mathbf{u}_{k, l}^{(a)}{ }^{\mathrm{H}} \mathbf{M}_{k}^{(a)} \mathbf{u}_{k, l}^{(a)},
\end{aligned}
$$

where $\mathbf{M}_{k}^{(a)}$ is the received signal covariance matrix for UE $k$. This is given for DL UE $k$ as

$$
\begin{aligned}
\mathbf{M}_{k}^{(\mathrm{dl})}= & \mathbb{E}_{d}\left[\mathbf{x}_{k}^{(\mathrm{dl})} \mathbf{x}_{k}^{(\mathrm{dll})^{\mathrm{H}}}\right]=\sum_{\{j, l\} \in \mathcal{A}_{\mathrm{dl}}} \mathbf{H}_{i_{j}, k}^{(\mathrm{dl})} \mathbf{m}_{j, l}^{(\mathrm{dl})}\left(\mathbf{H}_{i_{j}, k}^{(\mathrm{dl})} \mathbf{m}_{j, l}^{(\mathrm{dl})}\right)^{\mathrm{H}} \\
& +\sum_{\{j, l\} \in \mathcal{A}_{\mathrm{ul}}} \mathbf{H}_{j, k}^{(\mathrm{ul}-\mathrm{dl})} \mathbf{m}_{j, l}^{(\mathrm{ul})}\left(\mathbf{H}_{j, k}^{(\mathrm{ul}-\mathrm{dl})} \mathbf{m}_{j, l}^{(\mathrm{ul})}\right)^{\mathrm{H}}+\mathcal{N}_{0} \mathbf{I}
\end{aligned}
$$

and for UL UE $k$ at BS $i_{k}$ (note that in UL, the $\mathbf{M}_{k}^{(\mathrm{ul})}$ is the same for all $k \in \mathcal{U}_{i_{k}}$ ) as

$$
\begin{aligned}
\mathbf{M}_{k}^{(\mathrm{ul})} & =\mathbb{E}_{d}\left[\mathbf{x}_{i_{k}}^{(\mathrm{ul})} \mathbf{x}_{i_{k}}^{(\mathrm{ull})^{\mathrm{H}}}\right]=\sum_{\{j, l\} \in \mathcal{A}_{\mathrm{ul}}} \mathbf{H}_{i_{k}, j}^{(\mathrm{ul})} \mathbf{m}_{j, l}^{(\mathrm{ul})}\left(\mathbf{H}_{i_{k}, j}^{(\mathrm{ul})} \mathbf{m}_{j, l}^{(\mathrm{ul})}\right)^{\mathrm{H}} \\
& +\sum_{\{j, l\} \in \mathcal{A}_{\mathrm{dl}}} \mathbf{H}_{i_{j}, i_{k}}^{(\mathrm{dll})} \mathbf{m}_{j, l}^{(\mathrm{dl})}\left(\mathbf{H}_{i_{j}, i_{k}}^{(\mathrm{dl}-\mathrm{ul})} \mathbf{m}_{j, l}^{(\mathrm{dl})}\right)^{\mathrm{H}}+\mathcal{N}_{0} \mathbf{I} .
\end{aligned}
$$

The linear minimum MSE (MMSE) receiver employed for data detection can be obtained from (3) as

$$
\tilde{\mathbf{u}}_{k, l}^{(a)}=\left(\mathbf{M}_{k}^{(a)}\right)^{-1} \mathbf{H}_{i_{k}, k}^{(a)} \mathbf{m}_{k, l}^{(a)} .
$$

When the MMSE receiver is employed, the corresponding MSE can be obtain as

$$
\tilde{\epsilon}_{k, l}^{(a)}=1-\tilde{\mathbf{u}}_{k, l}^{(a)} \mathrm{H}_{\mathbf{H}} \mathbf{H}_{i_{k}, k}^{(a)} \mathbf{m}_{k, l}^{(a)},
$$

${ }^{2} \mathrm{RX}$ is used to refer DL UE for DL transmission or UL BS for UL transmission.

${ }^{3} \mathbb{E}_{d}\{$.$\} is the expectation operation over transmit data symbols.$ which is called the MMSE value of the estimated received data corresponding to the UL/DL data transmission of user $k$ from spatial stream $l$. Finally, assuming independent detection of data streams, we can write the signal-to-interference-plusnoise ratio (SINR) $\gamma_{k, l}^{(a)}$ at RX node for DL/UL data transmission as in (8) and (9).

\section{Decentralized BeAmformer Design}

The main problem in centralized dynamic TDD beamformer design is to acquire the CSI at the centralized node. That is, all the DL BS-UE channels, UL BS-UE channels, cross-UE channels and cross-BS channels should be available at the centralized unit. This is a tedious task as there are lots of cross-UE channels to be estimated and reported. Hence, decentralized beamformer design is more suited for dynamic TDD networks, where we can overcome the channel estimation problem by using bi-directional OTA signaling framework [18]. In this section, we study iterative decentralized beamformer design for dynamic TDD. First, we generalize the WQM solution from [27] to cover the dynamic TDD setting. Furthermore, we reformulate the problem for improved convergence using the ideas from [26] that were originally applied to WSR maximization. Second, we consider some special cases of WQM to obtain a beamformer design for QWSR maximization and SMSE minimization. In this Section, we consider only the algorithmic solution for the distributed framework. Practical implementation and related imperfections are presented in latter sections.

\section{A. Weighted Queue Minimization}

Here, weighted $\ell_{q}$-norm queue minimization of the UL and DL users is considered with sum transmit power constraints at the transmitters. Let $Q_{k}^{(\mathrm{dl})}$ denote the number of queued packets destined for DL user $k$ and $Q_{k}^{(\mathrm{ul})}$ denote the number of queued packets at UL user $k$ at a given scheduling instant. Also, we model the traffic generation in the network using Poisson arrival process, where $\lambda_{k}^{(\mathrm{dl})}(\tau) \sim \operatorname{Pois}\left(A_{k}^{(\mathrm{dl})}\right)$ is the generated traffic for DL user $k$ in time instance $\tau$. Similarly, $\lambda_{k}^{(\mathrm{ul})}(\tau) \sim \operatorname{Pois}\left(A_{k}^{(\mathrm{ul})}\right)$ defines the generated traffic at UL user $k$ at time instance $\tau$. Here, $A_{k}^{(\mathrm{dl})}=\mathbb{E}_{\tau}\left\{\lambda_{k}^{(\mathrm{dl})}\right\}$ and $A_{k}^{(\mathrm{ul})}=$ $\mathbb{E}_{\tau}\left\{\lambda_{k}^{(\mathrm{ul})}\right\}$ are the average number of packet arrivals in bits for the corresponding UL/DL users. Then, the total number of queued packets at $(\tau+1)$ th time instant destined/available to user $k$ is given by

$$
Q_{k}^{(a)}(\tau+1)=\left[Q_{k}^{(a)}(\tau)-R_{k}^{(a)}(\tau)\right]^{+}+\lambda_{k}^{(a)}(\tau),
$$

where $[x]^{+} \triangleq \max \{x, 0\}$ and $R_{k}^{(a)}$ is the transmission rate to user $k$ with $a=\{\mathrm{UL}, \mathrm{DL}\}$. Here, $R_{k}^{(a)}=\sum_{l=1}^{L_{k}} R_{k, l}^{(a)}$, where $R_{k, l}^{(a)}$ denotes the number of transmitted bits over the $l$ th spatial stream to user $k$. For SINR $\gamma_{k, l}^{(a)}$, the maximum rate is bounded by $R_{k, l}^{(a)} \leq \log _{2}\left(1+\gamma_{k, l}^{(a)}\right)$. Now, we can define the queue deviation metric for the DL and UL as

$$
\Psi_{k}^{(a)}=Q_{k}^{(a)}-R_{k}^{(a)}=Q_{k}^{(a)}-\sum_{l=1}^{L_{k}} \log _{2}\left(1+\gamma_{k, l}^{(a)}\right) .
$$




$$
\begin{aligned}
\gamma_{k, l}^{(\mathrm{dl})}=\frac{\left|\mathbf{u}_{k, l}^{(\mathrm{dl})}{ }^{\mathrm{H}} \mathbf{H}_{i_{k}, k}^{(\mathrm{dl})} \mathbf{m}_{k, l}^{(\mathrm{dl})}\right|^{2}}{\mathcal{N}_{0}\left\|\mathbf{u}_{k, l}^{(\mathrm{dl})}\right\|^{2}+\sum_{\substack{\{j, n\} \in \mathcal{A}_{\mathrm{dl}} \\
\{j, n\} \neq\{k, l\}}}\left|\mathbf{u}_{k, l}^{(\mathrm{dl})^{\mathrm{H}}} \mathbf{H}_{i_{j}, k}^{(\mathrm{dl})} \mathbf{m}_{j, n}^{(\mathrm{dl})}\right|^{2}+\sum_{\{j, n\} \in \mathcal{A}_{\mathrm{ul}}}\left|\mathbf{u}_{k, l}^{(\mathrm{dl})^{\mathrm{H}}} \mathbf{H}_{j, k}^{(\mathrm{ul}-\mathrm{dl})} \mathbf{m}_{j, n}^{(\mathrm{ul})}\right|^{2}} \\
\gamma_{k, l}^{(\mathrm{ul})}=\frac{\left|\mathbf{u}_{k, l}^{(\mathrm{ul})^{\mathrm{H}}} \mathbf{H}_{i_{k}, k}^{(\mathrm{ul})} \mathbf{m}_{k, l}^{(\mathrm{ul})}\right|^{2}}{\mathcal{N}_{0}\left\|\mathbf{u}_{k, l}^{(\mathrm{ul})}\right\|^{2}+\sum_{\substack{\{j, n\} \in \mathcal{A}_{\mathrm{ul}} \\
\{j, n\} \neq\{k, l\}}}\left|\mathbf{u}_{k, l}^{(\mathrm{ul})^{\mathrm{H}}} \mathbf{H}_{i_{k}, j}^{(\mathrm{ul})} \mathbf{m}_{j, n}^{(\mathrm{ul})}\right|^{2}+\sum_{\{j, n\} \in \mathcal{A}_{\mathrm{dl}}}\left|\mathbf{u}_{k, l}^{(\mathrm{ul})^{\mathrm{H}}} \mathbf{H}_{i_{j}, i_{k}}^{(\mathrm{dl}-\mathrm{ul})} \mathbf{m}_{j, n}^{(\mathrm{dl})}\right|^{2}}
\end{aligned}
$$

In order to simplify the notation, let $\tilde{\boldsymbol{\Psi}}^{(a)}$ denote a vector with elements $\tilde{\Psi}_{k}^{(a)} \triangleq \alpha_{k}^{1 / q} \Psi_{k}^{(a)}$. Here, $\alpha_{k}$ is the weighting factor, used to prioritize users based on their corresponding QoS requirements. The WQM problem can be formulated as

$$
\begin{array}{cl}
\underset{\substack{\mathbf{m}_{k, l}^{(a)}, \mathbf{u}_{k, l}^{(a)} \\
\text { s. t. }}}{ } & \left\|\tilde{\boldsymbol{\Psi}}^{(\mathrm{dl})}\right\|_{q}+\left\|\tilde{\boldsymbol{\Psi}}^{(\mathrm{ul})}\right\|_{q} \\
& \sum_{k \in \mathcal{U}_{i}} \sum_{l=1}^{L_{k}}\left\|\mathbf{m}_{k, l}^{(\mathrm{dl})}\right\|^{2} \leq P_{i}^{(\mathrm{dl})} \quad \forall i \\
& \sum_{l=1}^{L_{k}}\left\|\mathbf{m}_{k, l}^{(\mathrm{ul})}\right\|^{2} \leq P_{k}^{(\mathrm{ul})} \quad \forall k,
\end{array}
$$

where optimization variables are transmit precoders $\mathbf{m}_{k, l}^{(a)}$ and receive combiners $\mathbf{u}_{k, l}^{(a)}$. Also, $P_{i}^{(d l)}$ and $P_{k}^{(u l)}$ are the maximum transmit powers available at the $i$-th DL BS and the $k$-th UL UE, respectively. Note that (12a) includes an implicit rate constraint $Q_{k}^{(a)}-\sum_{l=1}^{L_{k}} R_{k, l}^{(a)} \geq 0$ on the maximum number of transmitted bits for each user as governed by the number of backlogged packets [27]. The $\ell_{q}$ norm is used to provide a trade off between the fairness and sum queue minimization.

At RX, we employ MMSE receivers. Hence, we obtain the following relation between the MSE and the SINR [17]

$$
\tilde{\epsilon}_{k, l}^{(a)}=\left(1+\gamma_{k, l}^{(a)}\right)^{-1}
$$

Therefore, we can write the user-specific rates using the userspecific MSE values as $R_{k}^{(a)}=\sum_{l=1}^{L_{k}}-\log _{2}\left(\tilde{\epsilon}_{k, l}^{(a)}\right)$. Now, we introduce auxiliary MSE constraint as in [26] to (12) and rewrite the optimization problem as

$$
\begin{aligned}
& \min _{\substack{\mathbf{m}_{k, l}^{(a)}, \mathbf{u}_{k, l}^{(a)}, t_{k, l}^{(a)}}} \sum_{\substack{a \in \mathrm{UL}, \mathrm{DL}\} \\
\{.}} \sum_{k} \alpha_{k}\left(Q_{k}^{(a)}-\sum_{l=1}^{L_{k}} t_{k, l}^{(a)} \log _{2}(\beta)\right)^{q} \\
& \text { s. t. } \quad \epsilon_{k, l}^{(a)} \leq \beta^{-t_{k, l}^{(a)}}
\end{aligned}
$$$$
\text { (12b), (12c), }
$$

where $\beta$ is a predefined constant to adjust the approximation function such that $\beta>0$ [26]. By introducing these MSE constraints, our objective becomes a convex function of auxiliary variables $t_{k, l}^{(a)}$. However, the constraint (14b) is still in non-convex form. The non-convexity in (14b) can be handled iteratively by using the first-order Taylor series approximation similarly to [26]

$$
\beta^{-t_{k, l}^{(a)}}=-K_{1} t_{k, l}^{(a)}+K_{2},
$$

where $K_{1}=\beta^{-\bar{t}_{k, l}^{(a)}} \log (\beta)$ and $K_{2}=\beta^{-\bar{t}_{k, l}^{(a)}}+\bar{t}_{k, l}^{(a)} K_{1}$. Here, $\bar{t}_{k, l}^{(a)}$ is the point of approximation. By employing the above approximation for the MSE constraints, we can re-write the equivalent optimaization problem as

$$
\begin{aligned}
& \min _{\substack{\mathbf{m}_{k, l}^{(a)}, \mathbf{u}_{k, l}^{(a)} \\
t_{k, l}^{(a)}}} \sum_{\substack{a \in \in \\
\{\mathrm{UL}, \mathrm{DL}\}}} \sum_{k} \alpha_{k}\left(Q_{k}^{(a)}-\sum_{l=1}^{L_{k}} t_{k, l}^{(a)} \log _{2}(\beta)\right)^{q} \\
& \text { s. t. } 1-2 \Re\left(\mathbf{u}_{k, l}^{(a)}{ }^{\mathrm{H}} \mathbf{H}_{i_{k}, k}^{(a)} \mathbf{m}_{k, l}^{(a)}\right) \\
&+\mathbf{u}_{k, l}^{(a)} \mathbf{M}_{k}^{\mathrm{H}} \mathbf{u}_{k, l}^{(a)} \leq-K_{1} t_{k, l}^{(a)}+K_{2}
\end{aligned}
$$

(12b), (12c).

Finally, the optimization problem (16) can be solved using alternating optimization $(\mathrm{AO})$ between the transmit and receive beamformers. We adopt an approach based on the KKT optimality conditions [26], [27]. Due to the independent nature of the optimization variables, the solution can be decoupled to calculate at $\mathrm{RX}$ and $\mathrm{TX}^{4}$ nodes iteratively. Therefore, we define tasks for each coordinated nodes to implement this distributed iterative solution. For example, the RX node performs the task 'Receiver update' and the TX node performs the task 'Transmitter update' as mentioned below.

\section{Receiver update}

Here, we present the beamformer design steps that the RX node should be performed. To do that, all the transmit precoders should be made available at the RX node. We begin by fixing, the transmit precoders and solving for the receive beamformers and the other variables (auxiliary and dual). First, we calculate MMSE receivers $\mathbf{u}_{k, l}^{(a)}$ using (6). Next, the corresponding MSE $\epsilon_{k, l}^{(a)}$ is obtained from (7). Then, the MSE bounds can be solved with respect to fixed MSE $\epsilon_{k, l}^{(a)}$ as

$$
t_{k, l}^{(a)}=\bar{t}_{k, l}^{(a)}+\frac{1}{\log (\beta-\xi)}\left(1-\epsilon_{k, l}^{(a)} \bar{t}_{k, l}^{(a)}\right),
$$

${ }^{4} \mathrm{TX}$ is used to refer DL BS for DL transmission or UL UE for UL transmission. 
where $\bar{t}_{k, l}^{(a)}$ denotes $t_{k, l}^{(a)}$ from the previous iteration, $\xi=\frac{\beta-\theta}{n^{\varsigma}}$, $\zeta \in \mathcal{R}$ is chosen to be an approximately small constant and $\theta>1$. Here, the multiplier $\frac{1}{\log (\beta-\rho)}$ can be considered as a step size and $\xi$ is used for more aggressive convergence as detailed in [26]. Then, dual variables $\omega_{k, l}^{(a)}$ corresponding to (15) are obtained as

$$
\begin{aligned}
\omega_{k, l}^{(a)} & =(1-\rho) \bar{\omega}_{k, l}^{(a)} \\
& +\rho\left[\frac{\alpha_{k} q \log _{2}(\beta)}{K_{1}}\left(Q_{k}^{(a)}-\sum_{l=1}^{L_{k}} t_{k, l}^{(a)} \log _{2}(\beta)\right)^{(q-1)}\right]^{+}
\end{aligned}
$$

where $\bar{\omega}_{k, l}^{(a)}$ denotes fixed $\omega_{k, l}^{(a)}$ from the previous iteration. Here, $\rho \in(0,1)$ controls the convergence and is used to prevent overallocation. From here on, we refer to $\omega_{k, l}^{(a)}$ as user specific weight. The calculated MMSE receivers and user-specific weights information must be conveyed to /made available at coordinating TX nodes, in order to the perform 'Transmitter update' task.

\section{Transmitter update}

In the next step, we fix the MMSE receivers and solve for the transmit precoders. At this point, we assume all the MMSE receiver information and user-specific weights available at the TX node (to be detailed in Section IV). The transmit beamformers $\mathbf{m}_{k, l}^{(a)}$ can be derived from the first-order optimality conditions of (14) as

$$
\mathbf{m}_{k, l}^{(a)}=\left(\boldsymbol{\Phi}_{k}^{(a)}+\nu_{k}^{(a)} \mathbf{I}\right)^{-1} \omega_{k, l}^{(a)} \mathbf{H}_{i_{k}, k}^{(a)} \mathbf{u}_{k, l}^{\mathrm{H}},
$$

where $\boldsymbol{\Phi}_{k}^{(a)}$ and $\nu_{k}^{(a)}$ are the weighted transmit covariance matrix and the dual variable corresponding to power constraint at the transmiter (UL UE $k$ or DL BS $i_{k}$ ). For DL BS $i_{k}$, $\boldsymbol{\Phi}_{k}^{(\mathrm{dl})} \forall k \in \mathcal{U}_{i_{k}}$ is given by

$$
\begin{aligned}
\boldsymbol{\Phi}_{k}^{(\mathrm{dl})}= & \sum_{\{j, l\} \in \mathcal{A}_{\mathrm{dl}}} \omega_{j, l}^{(\mathrm{dl})} \mathbf{H}_{i_{k}, j}^{(\mathrm{dl})}{ }^{\mathrm{H}} \mathbf{u}_{j, l}^{(\mathrm{dl})}\left(\mathbf{H}_{i_{k}, j}^{(\mathrm{dl})}{ }^{\mathrm{H}} \mathbf{u}_{j, l}^{(\mathrm{dl})}\right)^{\mathrm{H}} \\
& +\sum_{\{j, l\} \in \mathcal{A}_{\mathrm{ul}}} \omega_{j, l}^{(\mathrm{ul})} \mathbf{H}_{i_{j}, i_{k}}^{(\mathrm{dl}-\mathrm{ul})^{\mathrm{H}}} \mathbf{u}_{j, l}^{(\mathrm{ul})}\left(\mathbf{H}_{i_{j}, i_{k}}^{(\mathrm{dl}-\mathrm{ul})^{\mathrm{H}}} \mathbf{u}_{j, l}^{(\mathrm{ul})}\right)^{\mathrm{H}} .
\end{aligned}
$$

Similarly, for UL user $k, \boldsymbol{\Phi}_{k}^{(\mathrm{ul})}$ is given by

$$
\begin{aligned}
\boldsymbol{\Phi}_{k}^{(\mathrm{ul})}= & \sum_{\{j, l\} \in \mathcal{A}_{\mathrm{ul}}} \omega_{j, l}^{(\mathrm{ul})} \mathbf{H}_{i_{j}, k}^{(\mathrm{ul})^{\mathrm{H}}} \mathbf{u}_{j, l}^{(\mathrm{ul})}\left(\mathbf{H}_{i_{j}, k}^{(\mathrm{ul})^{\mathrm{H}}} \mathbf{u}_{j, l}^{(\mathrm{ul})}\right)^{\mathrm{H}} \\
& +\sum_{\{j, l\} \in \mathcal{A}_{\mathrm{dl}}} \omega_{j, l}^{(\mathrm{dl})} \mathbf{H}_{j, k}^{(\mathrm{ul}-\mathrm{dl})^{\mathrm{H}}} \mathbf{u}_{j, l}^{(\mathrm{dl})}\left(\mathbf{H}_{j, k}^{(\mathrm{ul}-\mathrm{dl})^{\mathrm{H}}} \mathbf{u}_{j, l}^{(\mathrm{dl})}\right)^{\mathrm{H}} .
\end{aligned}
$$

The transmit beamformers can efficiently solved from (19) by bisection search over the dual variables $\nu_{k}^{(\mathrm{dl})}$ and $\nu_{k}^{(\mathrm{ul})}$ to satisfy the power constraints $\sum_{k \in \mathcal{U}_{i}} \sum_{l=1}^{L_{k}}\left\|\mathbf{m}_{k, l}^{(\mathrm{dl})}\right\|^{2} \leq P_{i}^{(\mathrm{dl})}$ and $\sum_{l=1}^{L_{k}}\left\|\mathbf{m}_{k, l}^{(\mathrm{ul})}\right\|^{2} \leq P_{k}^{(\mathrm{ul})}$, respectively.

Here, we employed alternating optimization approach to calculate the precoders and decoders. Hence, beamformers are not optimal as they are optimized for their fixed counterparts. Thus, we need to repeat the precoder/decoder optimization until the estimated beamformers and/or the optimization objective have converged. This procedure is summarized in Algorithm 1. Note that the centralized unit can get the same solution by considering this distributed implementation as independent subproblems, which can be solved in parallel. However, for dynamic TDD setting, we are more interested in the decentralized solution. Moreover, detailed signaling structure to facilitate the iterative algorithm is explained in Section IV.

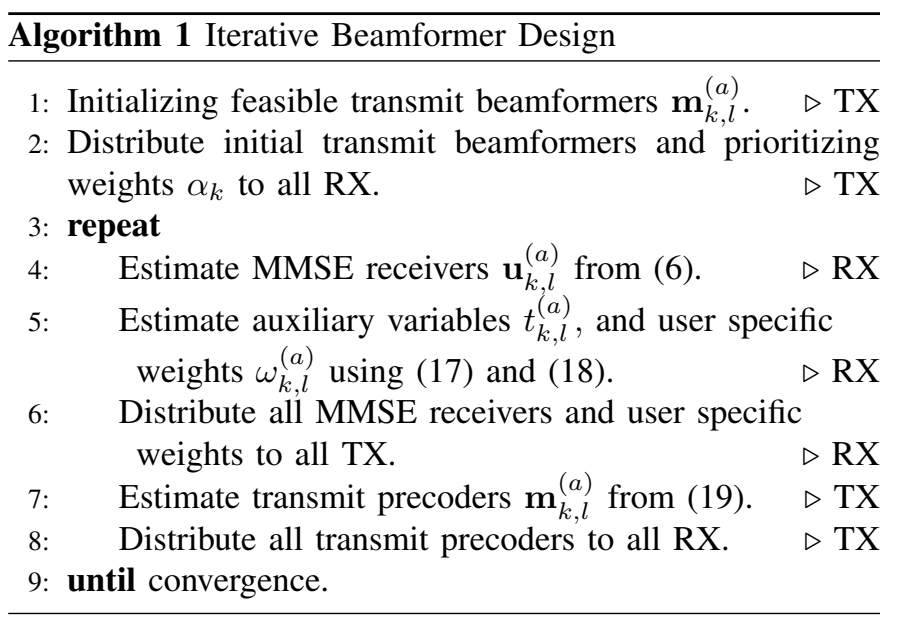

\section{B. Queue Weighted Sum Rate Maximization}

In this section, we consider a special case of WQM assuming $Q_{k}^{(a)}$ is large and $q=2$. Consequently, (14) is reduced to the following equivalent QWSR maximization problem

$$
\begin{aligned}
\max _{\substack{(a) \\
\mathbf{m}_{k, l}^{(a)}, \mathbf{u}_{k, l}^{(a)} \\
\text { s.t }}} & \sum_{\substack{a \in\{\mathrm{UL}, \mathrm{DL}\} \\
(12 \mathrm{~b}),(12 \mathrm{c}) .}} \sum_{k} \sum_{l=1}^{L_{k}} \alpha_{k} Q_{k}^{(a)} R_{k, l}^{(a)} \\
&
\end{aligned}
$$

We can follow the same procedure as in WQM to obtain an iterative beamformer design for the QWSR maximization problem. Since we assume queue size to be large, we do not need to control the over allocation of assigned rates as in (18). Therefore, user-specific weights $\omega_{k, l}^{(a)}$ are given simply as

$$
\omega_{k, l}^{(a)}=\frac{\alpha_{k} \log _{2}(\beta) Q_{k}^{(a)}}{K_{1}} .
$$

The other beamformer and auxiliary variable updates are the same as in WQM. Note that when $Q_{k}^{(a)}=1 \forall k$, QWSR objective is equivalent to WSR. Note that when $\beta=2$, the beamformer estimation procedure is equivalent to our previously studied WSMSE based approach [42]-[44].

\section{Sum MSE Minimization}

The WQM problem can be approximated by a simple SMSE minimizing beamformer design provided that the system operates in high SNR regime with equal user priorities, the queue sizes are large with $q=1$, and there are enough spatial degrees of freedom available such that all users can be served concurrently. The SMSE minimization problem can be formulated as

$$
\begin{aligned}
\min _{\mathbf{m}_{k, l}^{(a)} \mathbf{u}_{k, l}^{(a)}} & \sum_{\substack{a \in\{\mathrm{UL}, \mathrm{DL}\} \\
\text { s.t } \\
(12 \mathrm{~b}),(12 \mathrm{c}) .}} \sum_{k} \sum_{l=1}^{L_{k}} \epsilon_{k, l}^{(a)}
\end{aligned}
$$


In this special case, the weight updates are not needed and the optimal TX and RX beamformers (given fixed RX and TX beamformers) are solved directly from (19) and (6), respectively.

\section{TRAINING AND SignALING}

In the previous section, we considered iterative beamformer designs for WQM, QWSR, and SMSE objectives. In this section, we introduced possible approaches to implement these algorithms in practice. In all of the proposed iterative beamformer algorithms, we need to iteratively exchange or make available the intermediate beamformers and user-specific weights between the coordinated nodes. However, in dynamic TDD setup, we do not have the luxury to use a backhaul based approach as UEs are also playing a role in beamformer estimation. Hence, we employ F-B OTA training for the implicit information exchange [17], [18].

In the considered F-B OTA training scheme, we employ precoded (and weighted) pilot sequences in the forward and backward directions. Here, the forward direction refers to transmitters sending their training sequences, and the backward direction is referring to receivers sending their training sequences. The bi-directional training allows a fully distributed coordinated computation of transmit/receive beamformers without full CSI exchange over a backhaul. Furthermore, bi-directional signaling is embedded into each TDD frame to facilitate fast iterative information exchange as shown in Fig. 2 [18], [43].The nodes estimate their precoder/decoder based on the received forward/backward training sequences. Then, the estimated precoder/decoder is used for precoding the next iteration forward/backward training. Using this approach, we are able to practically implement the proposed beamformer designs.

It is possible to implement TDD frame structure in Fig. 2 (Scheduling block), with the approved 3GPP NR standard [9]. Beamformer signaling part can be implemented by concatenating multiple minislots together with the required guard period (GP) [18]. Then, multiple slots can be aggregated for data transmission. Moreover, each F-B iteration adds signaling overhead to the system. Hence, it is better to have a larger scheduling block to minimize the signaling overhead. However, it is constrained by coherence time and the traffic burstiness of the system. Note that, TDD frame should be synchronous over the UL/DL cells during the beamformer signaling. However, if the user scheduling remains the same for multiple TDD frames, and if the channel is slowly fading, the B-F training phase can use the beamformers from the previous frame as the starting point. Then, we can have less F-B iterations per TDD frame.

In a dense dynamic TDD network, a large number of orthogonal pilots would be required for ideal pilot estimation. In general, this is not possible due to the limited number of orthogonal resources. Therefore, we have to reuse the pilot sequences or employ non-orthogonal pilots during the training. Thus, the desired channel can be polluted by other user channels. This scenario is known as pilot contamination. Hence, it is essential to study the dynamic TDD system

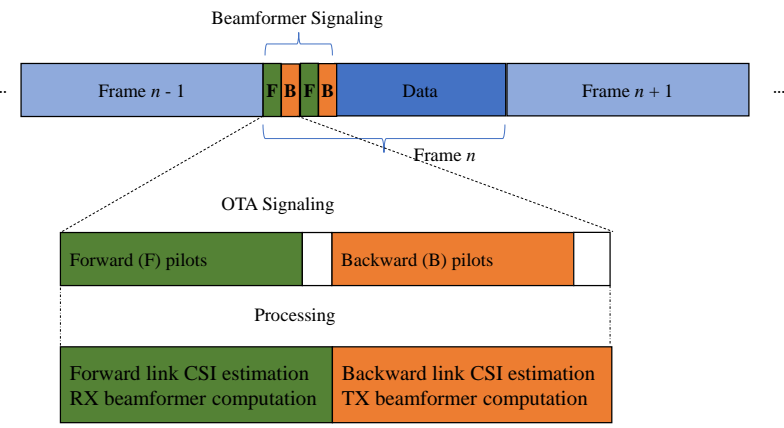

Fig. 2: TDD frame structure.

under imperfect OTA signaling conditions. Pilot contamination can be demoted in several ways, such as allocating pilots to minimize the pilot overlap and using clever channel estimation algorithms [36]-[40]. In the following subsections, we study various approaches to mitigate the pilot contamination while supporting the original beamformer design objectives. First, we consider three LS based strategies for direct beamformer estimation using only the precoded pilot information. Then, to further enhance the pilot decontamination, we propose pilot reuse method based on the large-scale fading information. Additionally, we analytically compare the proposed DE method with the SSE method to prove the superior decontamination capability of the DE approach.

\section{A. Pilot decontamination by direct beamformer estimation}

Here, we investigate the OTA signaling architecture under imperfect pilot conditions by assuming that the pilot training sequences used by each UE/BS are non-orthogonal, and only the pilot training sequences of the associated in-cell users are known to each UE/BS. As we employ non-orthogonal overlapping pilots, the effective channels are contaminated by interfering pilot training sequences. Therefore, they cannot be perfectly distinguished from each other. Without this information, we can not directly construct $\boldsymbol{\Phi}_{k}^{(\mathrm{dl})}$ in (20) at the DL BS $i_{k}$ or $\boldsymbol{\Phi}_{k}^{(\mathrm{ul})}$ in (21) at the UL UE $k$ in order to estimate their transmit precoders. To overcome this problem, we introduce three different strategies to facilitate the direct precoder/decoder estimation by using the received precoded training information directly without separately estimating all individual pilots (channels). In Table I, the signaling requirements for the forward/backward training are summarized for the proposed schemes. The proposed schemes are named as, Strategy A, Strategy B and Strategy C. Strategy A is proposed to implement exact beamformer design at both $\mathrm{RX}$ and $\mathrm{TX}$ with the minimum number OTA signaling (one forward and one backward training iteration per one beamformer signaling round). Hence, a separate (quantized) feedback link is required to transmit additional scalar weight information. Alternatively, we propose two designs; Strategy B and Strategy C. Both schemes use only the OTA signaling to exchange required information. In Strategy B, similar to Strategy A, we try to construct the beamformer design with the minimum number of OTA signaling. However, one backward training is not enough 
TABLE I: Precoded pilots/Feedback used in each Strategy.

\begin{tabular}{|l|l|l|l|l|}
\hline \multirow{2}{*}{ Strategy } & Forward & \multicolumn{2}{|c|}{ Backward Training } & \multirow{2}{*}{ Feedback } \\
\cline { 3 - 4 } & Training & 1st & 2nd & \\
\hline Strategy A & $\mathbf{m}_{k, l}^{(a)}$ & $\sqrt{\omega_{k, l}^{(a)}} \mathbf{u}_{k, l}^{(a)}$ & NA & $\sqrt{\omega_{k, l}^{(a)}}$ \\
Strategy B & $\mathbf{m}_{k, l}^{(a)}$ & $\mathbf{u}_{k, l}^{(a)}$ & NA & NA \\
Strategy C & $\mathbf{m}_{k, l}^{(a)}$ & $\sqrt{\omega_{k, l}^{(a)}} \mathbf{u}_{k, l}^{(a)}$ & $\omega_{k, l}^{(a)} \mathbf{u}_{k, l}^{(a)}$ & NA \\
\hline
\end{tabular}

to fully reconstruct the required weighted covariance matrix at the TX node. Hence, Strategy B follows an approximated beamformer design. To overcome the limitation of Strategy $\mathrm{B}$ and for the fully construction of the beamformer design, we propose Strategy $\mathrm{C}$ with two backward training resources. Further details on each strategy are presented in below.

\section{B. Strategy A}

In this strategy, we employ two training signals and one feedback message for each precoder/decoder iteration. First, forward pilots are transmitted by precoding the training sequence with the transmit beamformers $\mathbf{m}_{k, l}^{(a)}$. Then, the RXs estimate their MMSE receivers $\mathbf{u}_{k, l}^{(a)}$ and the corresponding user-specific weights $\omega_{k, l}^{(a)}$ by using the received pilot training matrices. Next, the RX nodes use weighted MMSE receivers $\sqrt{\omega_{k, l}^{(a)}} \mathbf{u}_{k, l}^{(a)}$ as the precoders for backward training pilots. At the same time, the RXs transmit square root of userspecific weights $\sqrt{\omega_{k, l}^{(a)}}$ through the feedback channel. Both, the backward pilot information and feedback information are used to estimate the transmit precoder $\mathbf{m}_{k, l}^{(a)}$ at TX node. In the following, the direct beamformer estimation procedure for Strategy A is described in more detail.

\section{Transmit Precoder Estimation}

Let $\mathbf{b}_{k, l} \in \mathbb{C}^{S}$ denote the pilot training sequence for $l^{\text {th }}$ data stream of DL/UL user $k$, where $S$ is the length of the pilot sequence. For backward training, the pilots are precoded with $\sqrt{\omega_{k, l}^{(a)}} \mathbf{u}_{k, l}^{(a)}$. Then, the received precoded pilot training matrix of user $k$ at DL BS $i_{k}$ (note that the $\mathbf{R}_{k}^{(\mathrm{dl})}$ is the same for all $\left.k \in \mathcal{U}_{i_{k}}\right)$ is given by

$$
\begin{aligned}
\mathbf{R}_{k}^{(\mathrm{dl})}= & \sum_{\{j, l\} \in \mathcal{A}_{\mathrm{dl}}} \sqrt{\omega_{j, l}^{(\mathrm{dl})}} \mathbf{H}_{i_{k}, j}^{(\mathrm{dl})}{ }^{\mathrm{H}} \mathbf{u}_{j, l}^{(\mathrm{dl})} \mathbf{b}_{j, l}^{\mathrm{H}} \\
& +\sum_{\{j, l\} \in \mathcal{A}_{\mathrm{ul}}} \sqrt{\omega_{j, l}^{(\mathrm{ul})}} \mathbf{H}_{i_{j}, i_{k}}^{(\mathrm{dl}-\mathrm{ul})^{\mathrm{H}}} \mathbf{u}_{j, l}^{(\mathrm{ul})} \mathbf{b}_{j, l}^{\mathrm{H}}+\mathbf{N}_{i_{k}}^{(\mathrm{dl})}
\end{aligned}
$$

where $\mathbf{N}_{i_{k}}^{(\mathrm{dl})} \in \mathbb{C}^{M_{i_{k}} \times S}$ is the estimation noise matrix for all pilot symbols. Similarly, the received pilot training matrix at UL user $k$, during the first backward training, is given by

$$
\begin{aligned}
\mathbf{R}_{k}^{(\mathrm{ul})}= & \sum_{\{j, l\} \in \mathcal{A}_{\mathrm{ul}}} \sqrt{\omega_{j, l}^{(\mathrm{ul})}} \mathbf{H}_{i_{j}, k}^{(\mathrm{ul})^{\mathrm{H}}} \mathbf{u}_{j, l}^{(\mathrm{ul})} \mathbf{b}_{j, l}^{\mathrm{H}} \\
& +\sum_{\{j, l\} \in \mathcal{A}_{\mathrm{dl}}} \sqrt{\omega_{j, l}^{(\mathrm{dl})}} \mathbf{H}_{j, k}^{(\mathrm{ul}-\mathrm{dl})^{\mathrm{H}}} \mathbf{u}_{j, l}^{(\mathrm{dl})} \mathbf{b}_{j, l}^{\mathrm{H}}+\mathbf{N}_{k}^{(\mathrm{ul})}
\end{aligned}
$$

where $\mathbf{N}_{k}^{(\mathrm{ul})} \in \mathbb{C}^{N_{k} \times S}$ is the estimation noise matrix.
At DL BS $i_{k}$, we recover a noisy version of $\boldsymbol{\Phi}_{k}^{(\mathrm{dl})} \forall k \in \mathcal{U}_{i_{k}}$ in (20) directly using the received composite channel information $\mathbf{R}_{k}^{(\mathrm{dl})}$ as,

$$
\overline{\mathbf{\Phi}}_{k}^{(\mathrm{dl})}=\mathbf{R}_{k}^{(\mathrm{dl})} \mathbf{R}_{k}^{(\mathrm{dl})^{\mathrm{H}}}=\boldsymbol{\Phi}_{k}^{(\mathrm{dl})}+\boldsymbol{\Omega}_{i_{k}},
$$

where $\boldsymbol{\Omega}_{i_{k}}$ includes all the cross-terms in (25) due to nonorthogonal pilots as well as noise. With orthogonal pilots, $\Omega_{i_{k}}$ would contain only estimation noise. The structure and significance of $\boldsymbol{\Omega}_{i_{k}}$ is examined in more detail in Section IV-E. Similarly, we can approximately construct $\boldsymbol{\Phi}_{k}^{(\mathrm{ul})}$ in (21) at UL user $k$ directly using the received composite channel information $\mathbf{R}_{k}^{(\mathrm{ul})}$ as

$$
\overline{\boldsymbol{\Phi}}_{k}^{(\mathrm{ul})}=\mathbf{R}_{k}^{(\mathrm{ul})} \mathbf{R}_{k}^{(\mathrm{ul})^{\mathrm{H}}}=\boldsymbol{\Phi}_{k}^{(\mathrm{ul})}+\boldsymbol{\Omega}_{k},
$$

where $\boldsymbol{\Omega}_{k}$ indicates the cross pilot interference.

With the knowledge of the received training matrices $\mathbf{R}_{k}^{(a)}$, user-specific weights $\sqrt{\omega_{k, l}^{(a)}}$ (received via the feedback channel) and own training sequences $\mathbf{b}_{k, l}$, we can locally estimate the transmit beamformers in a closed form expressions as

$$
\mathbf{m}_{k, l}^{(a)}=\left(\mathbf{R}_{k}^{(a)} \mathbf{R}_{k}^{(a)^{\mathrm{H}}}+\mathbf{I} \nu_{k}^{(a)}\right)^{-1} \sqrt{\omega_{k, l}^{(a)}} \mathbf{R}_{k}^{(a)} \mathbf{b}_{k, l},
$$

where the optimal $\nu_{k}^{(\mathrm{dl})}$ and $\nu_{k}^{(\mathrm{ul})}$ are found by bisection search to satisfy the power constraints $\sum_{k \in \mathcal{U}_{i}} \sum_{l=1}^{L_{k}}\left\|\mathbf{m}_{k, l}^{(\mathrm{dl})}\right\|^{2} \leq P_{i}^{(\mathrm{dl})}$ and $\sum_{l=1}^{L_{k}}\left\|\mathbf{m}_{k, l}^{(\mathrm{ul})}\right\|^{2} \leq P_{k}^{(\mathrm{ul})}$, respectively. In the ideal conditions with orthogonal pilots and very high pilot SNR (or very large $S$ ), (29) would be equal to (19).

\section{Receive Beamformer and Weights Estimation}

In the forward training, the pilots are precoded with the transmit precoders $\mathbf{m}_{k, l}^{(a)}$. Then, the received precoded pilot training matrix at DL user $k$ is given by,

$$
\begin{aligned}
\mathbf{T}_{k}^{(\mathrm{dl})}= & \sum_{\{j, l\} \in \mathcal{A}_{\mathrm{dl}}} \mathbf{H}_{i_{j}, k}^{(\mathrm{dl})} \mathbf{m}_{j, l}^{(\mathrm{dl})} \mathbf{b}_{j, l}^{\mathrm{H}} \\
& +\sum_{\{j, l\} \in \mathcal{A}_{\mathrm{ul}}} \mathbf{H}_{j, k}^{(\mathrm{ul}-\mathrm{dl})} \mathbf{m}_{j, l}^{(\mathrm{ul})} \mathbf{b}_{j, l}^{\mathrm{H}}+\mathbf{N}_{k}^{(\mathrm{dl})},
\end{aligned}
$$

where $\mathbf{N}_{k}^{(\mathrm{dl})} \in \mathbb{C}^{N_{k} \times S}$ is the estimation noise matrix for all pilot symbols. Similarly, the received precoded pilot training matrix at $\mathrm{UL} \mathrm{BS} i_{k}$ is given by

$$
\begin{aligned}
\mathbf{T}_{k}^{(\mathrm{ul})}= & \sum_{\{j, l\} \in \mathcal{A}_{\mathrm{ul}}} \mathbf{H}_{i_{k}, j}^{(\mathrm{ul})} \mathbf{m}_{j, l}^{(\mathrm{ul})} \mathbf{b}_{j, l}^{\mathrm{H}} \\
& +\sum_{\{j, l\} \in \mathcal{A}_{\mathrm{dl}}} \mathbf{H}_{i_{j}, i_{k}}^{(\mathrm{dl}-\mathrm{ul})} \mathbf{m}_{j, l}^{(\mathrm{dl})} \mathbf{b}_{j, l}^{\mathrm{H}}+\mathbf{N}_{i_{k}}^{(\mathrm{ul})},
\end{aligned}
$$

where $\mathbf{N}_{i_{k}}^{(\mathrm{ul})} \in \mathbb{C}^{M_{i_{k}} \times S}$ is the estimation noise matrix. Then, by using the received composite channel information $\mathbf{T}_{k}^{(a)}$ and own pilot training sequence $\mathbf{b}_{k, l}$ we can directly estimate the MMSE receivers as

$$
\mathbf{u}_{k, l}^{(a)}=\left(\mathbf{T}_{k}^{(a)} \mathbf{T}_{k}^{(a)^{\mathrm{H}}}+\mathcal{N}_{0} \mathbf{I}\right)^{-1} \mathbf{T}_{k}^{(a)} \mathbf{b}_{k, l}
$$

Also, the RX MSE can be estimated as

$$
\epsilon_{k, l}^{(a)}=1-\mathbf{u}_{k, l}^{(a)}{ }^{H} \mathbf{T}_{k}^{(a)} \mathbf{b}_{k, l} .
$$


Finally, we can estimate $\omega_{k, l}^{(a)}$ using (18) or (23) based on our optimization objective. Note that here we assume that, for any particular user, the forward and backward sequences are the same. However, the forward/backward pilots can also be designed separately.

\section{Strategy $B$}

In contrast to Strategy A, Strategy B does not require feedback channel to exchange the user-specific weights. Similar to Strategy A, the forward training pilots are precoded with the transmit precoders $\mathbf{m}_{k, l}^{(a)}$. Then, the RX nodes estimate the MMSE receivers $\mathbf{u}_{k, l}^{(a)}$. Next, the estimated MMSE receivers are used to precode the backward training pilots. The information received from the backward training pilots is enough to reconstruct the $\boldsymbol{\Phi}_{k}^{(a)}$ at TX $k / i_{k}$. However, by using locally evaluated user-specific weights, we can only approximately estimate the transmit precoders.

\section{Transmit Precoder Estimation}

In the backward training, the pilots are precoded with the MMSE receivers $\mathbf{u}_{k, l}^{(a)}$ without weights $\omega_{k, l}^{(a)}$. The received pilot training matrix at DL BS $i_{k}, \forall k \in \mathcal{U}_{i_{k}}$ is, then, given by

$$
\begin{aligned}
\mathbf{R}_{k, 1}^{(\mathrm{dl})}= & \sum_{\{j, l\} \in \mathcal{A}_{\mathrm{dl}}} \mathbf{H}_{i_{k}, j}^{(\mathrm{dl})^{\mathrm{H}}} \mathbf{u}_{j, l}^{(\mathrm{dl})} \mathbf{b}_{j, l}^{\mathrm{H}} \\
& +\sum_{\{j, l\} \in \mathcal{A}_{\mathrm{ul}}} \mathbf{H}_{i_{j}, i_{k}}^{(\mathrm{dl}-\mathrm{ul})^{\mathrm{H}}} \mathbf{u}_{j, l}^{(\mathrm{ul})} \mathbf{b}_{j, l}^{\mathrm{H}}+\mathbf{N}_{i_{k}}^{(\mathrm{dl})}
\end{aligned}
$$

Similarly, the received pilot training matrix at UL user $k$, during the first backward training, is given by

$$
\begin{aligned}
\mathbf{R}_{k, 1}^{(\mathrm{ul})}= & \sum_{\{j, l\} \in \mathcal{A}_{\mathrm{ul}}} \mathbf{H}_{i_{j}, k}^{(\mathrm{ul})^{\mathrm{H}}} \mathbf{u}_{j, l}^{(\mathrm{ul})} \mathbf{b}_{j, l}^{\mathrm{H}} \\
& +\sum_{\{j, l\} \in \mathcal{A}_{\mathrm{dl}}} \mathbf{H}_{j, k}^{(\mathrm{ul}-\mathrm{dl})^{\mathrm{H}}} \mathbf{u}_{j, l}^{(\mathrm{dl})} \mathbf{b}_{j, l}^{\mathrm{H}}+\mathbf{N}_{k}^{(\mathrm{ul})}
\end{aligned}
$$

Then, we can estimate the user weights $\omega_{k, l}^{(a)}$ locally by using the first set of received composite channel $\mathbf{R}_{k, 1}^{(a)}$ and training sequence $\mathbf{b}_{k, l}$ at TX $k / i_{k}$. To do that, first, we estimate MSE $\epsilon_{k, l}^{(a)}$ locally as

$$
\epsilon_{k, l}^{(a)}=1-\left(\mathbf{R}_{k, 1}^{(a)} \mathbf{b}_{k, l}\right)^{\mathrm{H}} \overline{\mathbf{m}}_{k, l}^{(a)}
$$

where $\overline{\mathbf{m}}_{k, l}^{(a)}$ is the transmit precoder estimated in the previous iteration or the initial condition. Then, we can estimate $\omega_{k, l}^{(a)}$ using (18) or (23). Finally, we construct $\overline{\mathbf{\Phi}}_{k, 1}^{(\mathrm{dl})}$, at DL BS $i_{k}$, as

$$
\begin{aligned}
\overline{\boldsymbol{\Phi}}_{k, 1}^{(\mathrm{dl}) \triangleq} & \mathbf{R}_{k, 1}^{(\mathrm{dl})}\left(\mathbf{I}+\sum_{j \in \mathcal{U}_{i_{k}}} \sum_{l=1}^{L_{j}} \frac{\left(\omega_{j, l}^{(\mathrm{dl})}-1\right)}{S} \mathbf{b}_{j, l} \mathbf{b}_{j, l}^{\mathrm{H}}\right) \mathbf{R}_{k, 1}^{(\mathrm{dl})}{ }^{\mathrm{H}} \\
= & \left.\sum_{j \in \mathcal{U}_{i_{k}}} \sum_{l=1}^{L_{j}} \omega_{j, l}^{(\mathrm{dl})} \mathbf{H}_{i_{k}, j}^{(\mathrm{dl})}{ }^{\mathrm{H}} \mathbf{u}_{j, l}^{(\mathrm{dl})}\left(\mathbf{H}_{i_{k}, j}^{(\mathrm{dl})} \mathbf{u}_{j, l}^{\mathrm{H}}\right)^{(\mathrm{dl})}\right)^{\mathrm{H}} \\
& +\sum_{\substack{\{j, l\} \in \mathcal{A}_{\mathrm{dl}} \\
j \notin \mathcal{U}_{i_{k}}}} \mathbf{H}_{i_{k}, j}^{(\mathrm{dl})}{ }^{\mathrm{H}} \mathbf{u}_{j, l}^{(\mathrm{dl})}\left(\mathbf{H}_{i_{k}, j}^{(\mathrm{dl})^{\mathrm{H}}} \mathbf{u}_{j, l}^{(\mathrm{dl})}\right)^{\mathrm{H}} \\
+ & \sum_{\{j, l\} \in \mathcal{A}_{\mathrm{ul}}} \mathbf{H}_{i_{j}, i_{k}}^{(\mathrm{dl}-\mathrm{ul})^{\mathrm{H}}} \mathbf{u}_{j, l}^{(\mathrm{ul})}\left(\mathbf{H}_{i_{j}, i_{k}}^{(\mathrm{dl}-\mathrm{ul})^{\mathrm{H}}} \mathbf{u}_{j, l}^{(\mathrm{ul})}\right)^{\mathrm{H}}+\boldsymbol{\Omega}_{i_{k}} .
\end{aligned}
$$

Similarly, at UL UE $k$, we construct $\overline{\boldsymbol{\Phi}}_{k}^{(\mathrm{ul})}$ as

$$
\begin{aligned}
\overline{\boldsymbol{\Phi}}_{k, 1}^{(\mathrm{ul}) \triangleq} & \mathbf{R}_{k, 1}^{(\mathrm{ul})}\left(\mathbf{I}+\sum_{l=1}^{L_{k}} \frac{\left(\omega_{k, l}^{(\mathrm{dl})}-1\right)}{S} \mathbf{b}_{k, l} \mathbf{b}_{k, l}^{\mathrm{H}}\right) \mathbf{R}_{k, 1}^{(\mathrm{ul})^{\mathrm{H}}} \\
= & \sum_{l=1}^{L_{k}} \omega_{k, l}^{(\mathrm{ul})} \mathbf{H}_{i_{k}, k}^{(\mathrm{ul})^{\mathrm{H}}} \mathbf{u}_{k, l}^{(\mathrm{ul})}\left(\mathbf{H}_{i_{k}, k}^{(\mathrm{ul})^{\mathrm{H}}} \mathbf{u}_{k, l}^{(\mathrm{ul})}\right)^{\mathrm{H}} \\
& +\sum_{\{j, l\} \in \mathcal{A}_{\mathrm{ul}}} \mathbf{H}_{i_{j}, k}^{(\mathrm{ul})^{\mathrm{H}}} \mathbf{u}_{j, l}^{(\mathrm{ul})}\left(\mathbf{H}_{i_{j}, k}^{(\mathrm{ul})^{\mathrm{H}}} \mathbf{u}_{j, l}^{(\mathrm{ul})}\right)^{\mathrm{H}} \\
+ & \sum_{\{j, l\} \in \mathcal{A}_{\mathrm{dl}}} \mathbf{H}_{j, k}^{(\mathrm{ul}-\mathrm{dl})^{\mathrm{H}}} \mathbf{u}_{j, l}^{(\mathrm{dl})}\left(\mathbf{H}_{j, k}^{(\mathrm{ul}-\mathrm{dl})^{\mathrm{H}}} \mathbf{u}_{j, l}^{(\mathrm{dl})}\right)^{\mathrm{H}}+\mathbf{\Omega}_{k} .
\end{aligned}
$$

It is clear that in this estimation scheme, $\overline{\boldsymbol{\Phi}}_{k, 1}^{(\mathrm{ul})}$ does not contain the ideal $\boldsymbol{\Phi}_{k}^{(a)}$ from (20)-(21) unlike in (37)-(38). This is mainly due to unavailability of the user specific weights from the interfered users. This is equivalent to assuming $\omega_{k, l}^{(a)}=1$ for all non-local data streams. As in Strategy A, with the knowledge of the received training matrices $\mathbf{R}_{k, 1}^{(a)}$ and own training sequences $\mathbf{b}_{k, l}$, we can estimate the transmit beamformers directly in closed form as

$$
\mathbf{m}_{k, l}^{(a)}=\left(\overline{\mathbf{\Phi}}_{k, 1}^{(a)}+\mathbf{I} \nu_{k}^{(a)}\right)^{-1} \omega_{k, l}^{(a)} \mathbf{R}_{k, 1}^{(a)} \mathbf{b}_{k, l}
$$

where the optimal $\nu_{k}^{(a)}$ is found by bisection search similarly to (29). The receive beamformer estimation procedure for Strategy B is same as in Strategy A.

\section{Strategy $C$}

In this strategy, we employ three training pilots per beamformer iteration, one in the forward direction and two consecutive training pilots in the backward direction. The forward training procedure is same as strategies $\mathrm{A}$ and $\mathrm{B}$, where we use transmit precoders $\mathbf{m}_{k, l}^{(a)}$ as the pilot precoders. The RXs estimate their MMSE receivers $\mathbf{u}_{k, l}^{(a)}$ and the corresponding user weights $\omega_{k, l}^{(a)}$ from the received pilots. In the backward phase, the first training sequences are precoded with $\sqrt{\omega_{k, l}^{(a)}} \mathbf{u}_{k, l}^{(a)}$ and the second transmission pilot sequences are precoded with $\omega_{k, l}^{(a)} \mathbf{u}_{k, l}^{(a)}$. The estimated pilot training matrices for the first pilot sequence are the same as in Strategy A (25)-(26) denoted 
by $\mathbf{R}_{k}^{(a)}$. The training matrix corresponding to the second pilot sequence denoted by $\mathbf{R}_{k, 2}^{(a)}$ is the same except the weight difference $\left(\sqrt{\omega_{k, l}^{(a)}}\right.$ replaced with $\left.\omega_{k, l}^{(a)}\right)$. The backward pilot information is used to estimate the transmit precoder $\mathbf{m}_{k, l}^{(a)}$ as

$$
\mathbf{m}_{k, l}^{(a)}=\left(\mathbf{R}_{k}^{(a)} \mathbf{R}_{k}^{(a)^{\mathrm{H}}}+\mathbf{I} \nu_{k}^{(a)}\right)^{-1} \mathbf{R}_{k, 2}^{(a)} \mathbf{b}_{k, l} .
$$

Other expressions are derived similarly to Strategies A and B and are omitted here to avoid repetition.

\section{E. DE vS SSE}

The signaling and training strategies proposed in previous subsections directly estimate the beamformers (DE approach) from the received pilot training matrices. As an alternative implementation, we consider the SSE technique. In the SSE method, we separately estimate each pilot, and the estimated information is used to calculate beamformers and user weights. Therefore, all the coordinating nodes need to know the pilot sequences used at each node. The SSE approach can be highly vulnerable to pilot contamination, if non-orthogonal pilot sequences are employed for OTA signaling. However, the $\mathrm{DE}$ is better suited for noisy environments and more resilient to pilot contamination. In this section, we analyze the DE and SSE estimation inaccuracies by considering the estimation of $\boldsymbol{\Phi}_{k}^{(\mathrm{dl})}$ in Strategy A. Also, without loss of generality, we examine $D L$ only case $\left(\mathcal{A}_{\mathrm{ul}}=\emptyset\right)$ in order to ease the notational complexity.

In stream specific estimation, we assume that all pilots are first individually estimated. Then, the covariance matrices and beamformers are constructed from those individual parts. The signaling model remains the same, that is, the received training matrix is of form (25). Recall that $\mathbf{R}_{k}^{(\mathrm{dl})}$ is the same for all $k \in \mathcal{U}_{i_{k}}$. From (25), each DL BS $i_{k}=1, \ldots, B, k \in \mathcal{U}_{i_{k}}$ estimate the UE (stream) specific pilots as

$$
\begin{aligned}
\mathbf{f}_{i_{k}, j, l}^{(\mathrm{dl})} & =\mathbf{R}_{k}^{(\mathrm{dl})} \mathbf{b}_{j, l}, \forall(j, l) \\
& =\sqrt{\omega_{j, l}^{(\mathrm{dl})}} \mathbf{H}_{i_{k}, j}^{(\mathrm{dl})}{ }^{\mathrm{H}} \mathbf{u}_{j, l}^{(\mathrm{dl})}+\boldsymbol{\delta}_{i_{k}, j, l},
\end{aligned}
$$

where $\boldsymbol{\delta}_{i_{k}, j, l}$ denotes the estimation noise and pilot contamination corresponding to pilot sequence $\mathbf{b}_{j, l}$ at BS $i_{k}$. We can immediately observe a potential downside to this. In order to accomplish (41), BS $i_{k}$ has to know all pilot sequences $\mathbf{b}_{j, l}$. Also, the amount of estimation noise and pilot contamination gradually increase with the number of pilot sequences. Then using all the collected stream specific estimates, the matrix $\left(\overline{\boldsymbol{\Phi}}_{k}^{(\mathrm{dl})}\right)_{\text {SSE }}$ for the SSE approach is given by

$$
\begin{aligned}
\left(\overline{\mathbf{\Phi}}_{k}^{(\mathrm{dl})}\right)_{\mathrm{SSE}}= & \sum_{\{j, l\} \in \mathcal{A}_{\mathrm{dl}}} \mathbf{f}_{i_{k}, j, l}^{(\mathrm{dl})} \mathbf{f}_{i_{k}, j, l}^{(\mathrm{dl})}=\boldsymbol{\Phi}_{k}^{\mathrm{H}}+\mathbf{K}_{k} \\
& +\overline{\mathbf{K}}_{k}+\sum_{\{j, l\} \in \mathcal{A}_{\mathrm{dl}}} \mathbf{N}_{i_{k}}^{(\mathrm{dll})} \mathbf{b}_{j, l} \mathbf{b}_{j, l}^{\mathrm{H}} \mathbf{N}_{i_{k}}^{(\mathrm{dll})^{\mathrm{H}}}
\end{aligned}
$$

where $\mathbf{K}_{k}$ and $\overline{\mathbf{K}}_{k}$ are additional interference terms given in (43) and (44), respectively. On the other hand, the estimation expressions $\left(\overline{\boldsymbol{\Phi}}_{k}^{(\mathrm{dl})}\right)_{\mathrm{DE}}$ for the DE approach are given by

$$
\left(\overline{\boldsymbol{\Phi}}_{k}^{(\mathrm{dl})}\right)_{\mathrm{DE}}=\mathbf{R}_{k}^{(\mathrm{dl})} \mathbf{R}_{k}^{(\mathrm{dl}) \mathrm{H}}=\boldsymbol{\Phi}_{k}^{(\mathrm{dl})}+\mathbf{K}_{k}+\mathbf{N}_{i_{k}}^{(\mathrm{dl})} \mathbf{N}_{i_{k}}^{(\mathrm{dl})^{\mathrm{H}}}
$$

From the expressions in (45) and (42), we can observe that SSE expression differs from DE case due to additional estimation error term $\overline{\mathbf{K}}_{k}$ and estimated noise terms $\mathbf{N}_{i_{k}}^{(\mathrm{dl})} \mathbf{N}_{i_{k}}^{(\mathrm{dl})}{ }^{\mathrm{H}}$ and $\sum_{\{j, l\} \in \mathcal{A}_{\mathrm{dl}}} \mathbf{N}_{i_{k}}^{(\mathrm{dl})} \mathbf{b}_{j, l} \mathbf{b}_{j, l}^{\mathrm{H}} \mathbf{N}_{i_{k}}^{(\mathrm{dll})}{ }^{\mathrm{H}}$. In order to compare DE and SSE approaches, first, we can see that $\mathbb{E}\left[\mathbf{N}_{i_{k}}^{(\mathrm{dl})} \mathbf{N}_{i_{k}}^{(\mathrm{dl})}{ }^{\mathrm{H}}\right]$ is a diagonal matrix of size $M_{i_{k}} \times M_{i_{k}}$ with each diagonal element equal to $\mathcal{N}_{0} S$, i.e.,

$$
\operatorname{Tr}\left(\mathbb{E}\left[\mathbf{N}_{i_{k}}^{(\mathrm{dl})} \mathbf{N}_{i_{k}}^{(\mathrm{dl})}{ }^{\mathrm{H}}\right]\right)=M_{i_{k}} \mathcal{N}_{0} S .
$$

For the SSE case, we can form a symmetric matrix $\mathbf{C}_{i_{k}}=$ $\sum_{\{j, l\} \in \mathcal{A}_{\mathrm{dl}}} \mathbf{b}_{j, l} \mathbf{b}_{j, l}^{\mathrm{H}}$ with the size of $S \times S$, where the diagonal terms are equal to $K / S$. Since the matrix trace is invariant under cyclic permutations, the following holds

$$
\operatorname{Tr}\left(\mathbb{E}\left[\sum_{\{j, l\} \in \mathcal{A}_{\mathrm{dl}}} \mathbf{N}_{i_{k}}^{(\mathrm{dl})} \mathbf{b}_{j, l} \mathbf{b}_{j, l}^{\mathrm{H}} \mathbf{N}_{i_{k}}^{\left(\mathrm{dll}{ }^{\mathrm{H}}\right.}\right]\right)=M_{i_{k}} \mathcal{N}_{0} K .
$$

Assuming a practical scenario where the number of users served is larger than the available pilot resources (sequence length), $K>S$, then

$$
\operatorname{Tr}\left(\mathbb{E}\left[\sum_{\{j, l\} \in \mathcal{A}_{\mathrm{dl}}} \mathbf{N}_{i_{k}}^{(\mathrm{dll})} \mathbf{b}_{j, l} \mathbf{b}_{j, l}^{\mathrm{H}} \mathbf{N}_{i_{k}}^{(\mathrm{dll})^{\mathrm{H}}}\right]\right)>\operatorname{Tr}\left(\mathbb{E}\left[\mathrm{N}_{i_{k}}^{(\mathrm{dll})} \mathbf{N}_{i_{k}}^{(\mathrm{dll})^{\mathrm{H}}}\right]\right) .
$$

Finally, by taking the MSE of estimates (45) and (42), we can obtain the following relationship

$$
\mathbb{E}\left[\left|\left(\boldsymbol{\Phi}_{k}^{(\mathrm{dl})}\right)_{\mathrm{SSE}}-\boldsymbol{\Phi}_{k}^{(\mathrm{dl})}\right|^{2}\right] \succ \mathbb{E}\left[\left|\left(\boldsymbol{\Phi}_{k}^{(\mathrm{dl})}\right)_{\mathrm{DE}}-\boldsymbol{\Phi}_{k}^{(\mathrm{dl})}\right|^{2}\right]+\overline{\mathbf{K}}_{k} \overline{\mathbf{K}}_{k}^{\mathrm{H}},
$$

where the inequality follows from (48) and from the fact that $\overline{\mathbf{K}}_{k} \overline{\mathbf{K}}_{k}^{\mathrm{H}}$ is a positive definite matrix. Thus, DE provides better estimation performance than SSE in the MSE sense.

\section{F. Decontamination via Pilot Reuse}

In this section, we consider a traditional pilot reuse approach to enhance the pilot decontamination further. The main aim is to assess how much additional gain can be achieved by applying centralized pilot assignment as compared to the decentralized direct estimation methods proposed in Section IV. A simple pilot reuse approach is proposed for complicated dynamic TDD setup based only on large-scale fading information of the BS-UE and UE-UE channels. In dynamic TDD networks, finding a proper utility for the pilot assignment is challenging. For example, the received channel information at DL UE $k$ in the forward training phase and received channel information at DL BS $i_{k}$ in the backward training phase are contaminated with different set of BSs and UEs. In our previous work, we introduced logarithmically weighted interference-to-signal-ratio (ISR) based decontamination method for DL only case [45]. Here, we use the same approach but with slight modifications to cope with specific challenges due to the dynamic TDD scenario.

In the considered heuristic method, the pilot allocation is carried out utilizing the user specific path loss measurements and reports. The basic idea is to reuse the same pilot for two users when they are physically far from each other. In 


$$
\begin{aligned}
& \mathbf{K}_{k}=\sum_{\substack{\{j, l\} \in \mathcal{A}_{\mathrm{dl}} \\
\{y, z\} \in \mathcal{A}_{\mathrm{dl}} \\
(y \neq j \& z \neq l)}} \sqrt{\omega_{j, l}^{(\mathrm{dl})} \omega_{y, z}^{(\mathrm{dl})}} \mathbf{H}_{i_{k}, j}^{(\mathrm{dl})} \mathbf{u}_{j, l}^{\mathrm{H}} \mathbf{b}_{j, l}^{\mathrm{d} l} \mathbf{b}_{y, z}\left(\mathbf{H}_{i_{k}, y}^{(\mathrm{dl})^{\mathrm{H}}} \mathbf{u}_{y, z}^{(\mathrm{dl})}\right)^{\mathrm{H}}+\sum_{\{j, l\} \in \mathcal{A}_{\mathrm{dl}}} \sqrt{\omega_{j, l}^{(\mathrm{dl})}}\left(\mathbf{H}_{i_{k}, j}^{(\mathrm{dl})^{\mathrm{H}}} \mathbf{u}_{j, l}^{(\mathrm{dl})} \mathbf{b}_{j, l}^{\mathrm{H}} \mathbf{N}_{i_{k}}^{(\mathrm{dl})^{\mathrm{H}}}+\mathbf{N}_{i_{k}}^{(\mathrm{dl})} \mathbf{b}_{j, l}\left(\mathbf{H}_{i_{k}, j}^{(\mathrm{dl})} \mathbf{u}_{j, l}^{\mathrm{H}}\right)^{(\mathrm{dl})}\right) \\
& \overline{\mathbf{K}}_{k}=\sum_{\substack{\{\{j, l\},\{y, z\},\{s, t\}\} \in \mathcal{A}_{\mathrm{dl}} \\
(s \neq j \& t \neq l)}} \sqrt{\omega_{s, t}^{(\mathrm{dl})} \omega_{y, z}^{(\mathrm{dl})}} \mathbf{H}_{i_{k}, s}^{(\mathrm{dl})} \mathbf{u}_{s, t}^{\mathrm{H}} \mathbf{b}_{s, t}^{\mathrm{H}} \mathbf{b}_{j, l} \mathbf{b}_{j, l}^{\mathrm{H}} \mathbf{b}_{y, z}\left(\mathbf{H}_{i_{k}, y}^{(\mathrm{dl})} \mathbf{u}_{y, z}^{\mathrm{H}}\right)^{\mathrm{H}} \\
& +\sum_{\substack{\{j, l\},\{y, z\}\} \in \mathcal{A}_{\mathrm{dl}} \\
(y \neq j \& z \neq l)}} \sqrt{\omega_{y, z}^{(\mathrm{dl})}}\left(\mathbf{H}_{i_{k}, y}^{(\mathrm{dl})^{\mathrm{H}}} \mathbf{u}_{y, z}^{(\mathrm{dl})} \mathbf{b}_{y, z}^{\mathrm{H}} \mathbf{b}_{j, l} \mathbf{b}_{j, l}^{\mathrm{H}} \mathbf{N}_{i_{k}}^{(\mathrm{dl})^{\mathrm{H}}}+\mathbf{N}_{i_{k}}^{(\mathrm{dl})} \mathbf{b}_{j, l} \mathbf{b}_{j, l}^{\mathrm{H}} \mathbf{b}_{y, z}\left(\mathbf{H}_{i_{k}, y}^{(\mathrm{dl})^{\mathrm{H}}} \mathbf{u}_{y, z}^{(\mathrm{dl})}\right)^{\mathrm{H}}\right)
\end{aligned}
$$

addition to BS-UE path loss measurements, we assume UE-UE received signal strength indicator (RSSI) based measurements are made available at BSs to avoid allocating same pilots to severely interfering DL and UL users. In order to do that, UE should be able to listen to received signals from both BSs and nearby UEs. Based on the RSSI measurements, UE reports a set of strongest measured RSSI values, both from BSs and UEs to serving BS via a separate control channel.

To assign the pilots, we define a cost function $R_{\mathcal{G}_{p}}$ for every orthogonal pilot $p \in\{1, \ldots, S\}$ that is shared with a set of users $\mathcal{G}_{p} \subset \mathcal{U}$ as

$$
R_{\mathcal{G}_{p}}=\sum_{k \in \mathcal{G}_{p}} \log \left(1+\sum_{j \in \mathcal{G}_{p} /\{k\}} I_{j} / S_{k}\right)
$$

where $S_{k}$ is the path gain between user $k$ serving BS $i_{k}$, and $I_{j}$ is the path gain between interfering user $j$ and BS $i_{k}$. Logarithmic ISR weighting is used to provide fairness such that weak users (far from BS) are not severely penalized. Every time a pilot is reused the cost function is increased correspondingly. The resulting ISR value is scaled by 1 to make the utility function always positive. Finally, the minimization problem used for the pilot allocation is formulated as

$$
\begin{array}{ll}
\underset{\mathcal{G}_{p} \forall p}{\operatorname{minimize}} & \sum_{p=1}^{S} R_{\mathcal{G}_{p}} \\
\text { s.t } \quad \mathcal{Y}_{k} & \cap \mathcal{G}_{p}=\emptyset \quad \forall k, p,
\end{array}
$$

where $\mathcal{Y}_{k}$ is the set of users with shortest UE-UE distance to user $k$. Finding optimal solution for above integer problem is highly complex for large $S$ and $K$. Hence, a sub-optimal greedy method is used to solve the problem and the entire process is summarized in Algorithm 2. First, potential cost values for each pair are computed assuming they share the same pilot. Then, the user pair with minimum cost is assigned with the same pilot. Consequently, next pilot resources are assigned to the unassigned user pairs with the minimum utility value. Continuing this, up to $2 S$ users are assigned. Then, the rest of the unassigned users are allocated to pilots using the same greedy method on the per pilot basis such a way that corresponding pilot will not be used more than $K / S+1$ times. Here, the main target is to minimize the total log weighted pilot interference in the system. This may not accurately match with the original traffic aware optimization objective in Section III. However, the results show significant sum rate improvement as compared to random pilot assignment.

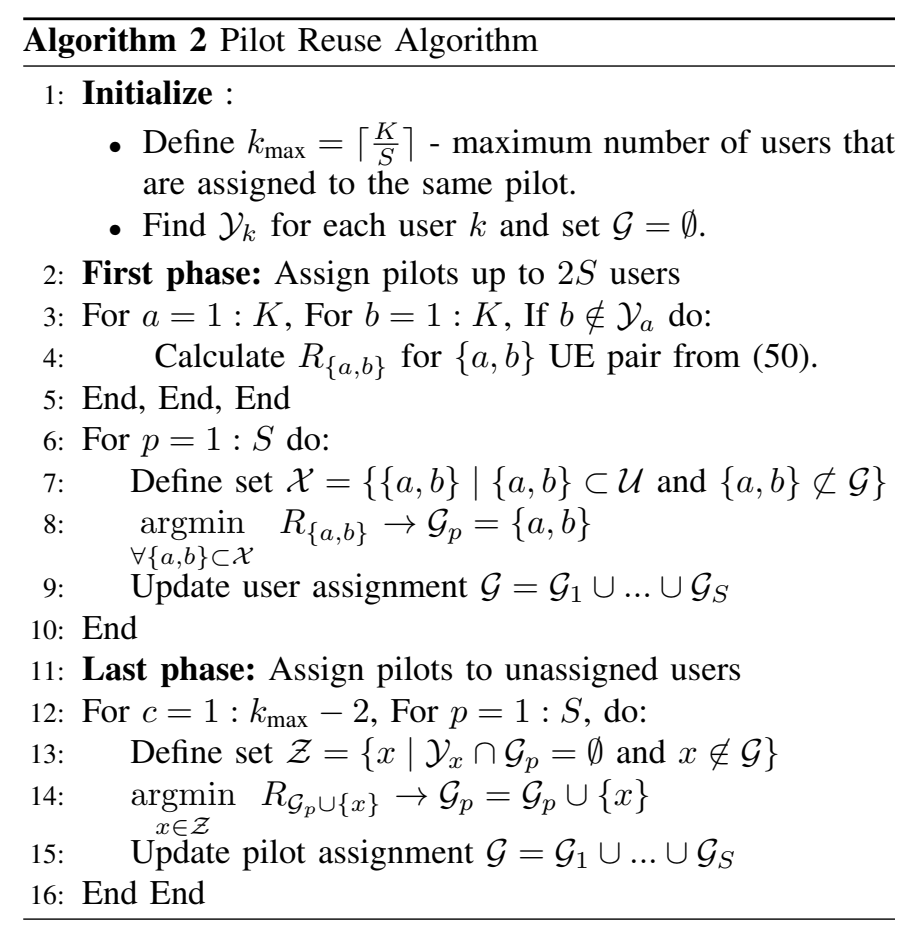

\section{G. Complexity and Overhead}

It is important to study the complexity and associated overhead of the proposed DE-based strategies. Therefore, in this subsection, we look into those two concerns.

\section{Complexity Study}

Here, we study the computational complexity of the proposed direct beamformer estimation methods. All strategies have similar computational complexities. Hence, we consider Strategy A in this study. In proposed iterative strategies, the complexity is linearly proportional to the number of OTA signaling rounds. Hence, we focus on one iteration only. There are 4 types of nodes involved in computing beamformers. The corresponding complexity at each node is as follows,

- DL BS - The dominant operation is the matrix inversion in (29). Also, there is a dual variable which we found by 
bisection search to satisfy the power constraint. Hence, the complexity is $\mathcal{O}\left(M_{i}^{3} \times \Delta \times K_{i} \times L_{k}\right)$, where $\Delta$ is the number bisection iterations required to satisfy the power constraint.

- DL UE - Here, the dominant operation is the matrix product operation within the inverse matrix in (32). Hence the complexity is $\mathcal{O}\left(N_{k}^{2} \times S\right)$.

- UL BS - The complexity due to operations in (32) is roughly $\mathcal{O}\left(M_{i}^{2} \times\left(M_{i}+S+L_{k} \times K_{i}\right)\right)$.

- UL UE - The dominant complexity is coming from the bisection iterations and the matrix inversion in (29). Therefore, the complexity is $\mathcal{O}\left(N_{k}^{3} \times \Delta \times L_{k}\right)$.

It is obvious that BS should have a higher computational capability compared to UEs. For the proposed strategies, for BSs, maximum complexity requirement arise when it is in DL mode. Which is $\mathcal{O}\left(M_{i}^{3} \times \Delta \times K \times L_{k}\right)$ per one beamformer signaling round. Also, for UEs, maximum complexity requirement is $\mathcal{O}\left(N_{k}^{3} \times \Delta \times L_{k}\right)$, which arises when it is in UL mode.

\section{Overhead Model}

As in Fig.2, the TDD frame is divided into two portions; 1. beamformer signalling, 2 . data transmission. Therefore, the actual achievable rate of the system pretty much depends on the beamformer training duration. Here, we can model it as,

$$
R_{\mathrm{ac}}=\left(1-\frac{\tau_{0}}{T} \mathrm{BIT}\right) R_{\mathrm{sum}},
$$

where, $R_{\text {ac }}$ is the actual achievable sum rate and $R_{\text {sum }}$ is the achieved sum rate from the iterative algorithm after BIT bidirectional precoder/decoder training rounds. The signaling overhead per one signaling round is $\frac{\tau_{0}}{\mathrm{~T}}$, where $\tau_{0}$ and $T$ are the duration for a one precoder/decoder iteration and the duration of the TDD frame, respectively. Here, we refer $\tau_{0}$ as the 'effective overhead duration per F-B training round'. Following tasks are performed during the $\tau_{0}$,

- Tx nodes transmit precoded pilots, which are precoded with transmit beamformers (F-training).

- Rx nodes estimate receiver beamformers and user-specific weights.

- Rx nodes transmit pilots using the estimated receive beamformers as pilot precoders possibly weighted by the user-specific weights as described in Section IV.B-D (Btraining). Moreover, in Strategy A, we utilize a separate (quantized) feedback mechanism to exchange the scalar weights.

- Tx nodes estimate transmit precoders.

In addition to the above tasks, a guard period is required when the communication direction changes forward to backward and vice-versa. However, the overhead related for exchanging control messages such as measured RSSI values, explicit information about the queue sizes and prioritize coefficients is not included in this formula as the reporting period for such messages is much longer. In numerical examples, we investigate optimal number of bi-directional signaling rounds required for a TDD frame with this overhead model.

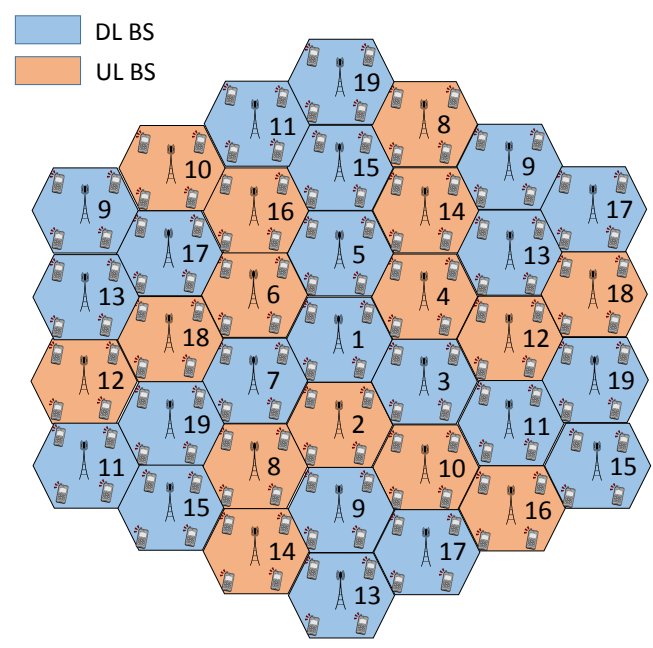

Fig. 3: 19-cell wrap-up model.

\section{NumericAl EXAMPLES}

The simulations are carried out for nineteen cell $(=19)$ wrap-around model as illustrated in Fig. 3. The distances between the BSs are considered to be $200 \mathrm{~m}$ and the UEs are randomly placed at the cell edge. For all the simulations, the path loss exponent is fixed to 3.67. The power constraint for DL BSs and UL UEs are normalized such that $P_{k}^{(\mathrm{ul})}=$ $P_{i}^{(\mathrm{dl})} / K_{i}$, i.e., the total cell-specific UL power is equal to the DL power. However, we consider different power allocation in Fig. 7. Moreover, SNR is defined with respect to the DL cell edge user ( $\mathrm{SNR}=S_{k} P_{i_{k}}^{(\mathrm{dl})} / \mathcal{N}_{0}$ ). To simplifying the study, all the user priority weights are assumed to be $\alpha_{k}=1$. In Fig. 8, we examine time-correlated block fading, which is generated using Jake's Doppler spectrum model with the normalized user terminal velocity $t_{\mathrm{S}} f_{\mathrm{D}}=0.01$, where $t_{\mathrm{S}}$ and $f_{\mathrm{D}}$ are the signaling rate and the maximum Doppler shift, respectively. For the rest of the figures, we have used uncorrelated block fading model. We have randomly assigned UL/DL BSs with DL cell probability $=0.5$, for all the results except in Fig. 6 . User weights $\alpha_{k} Q_{k}^{(a)}=1 \forall k$ are used for all results with WSR maximization. Perfect CSIT is assumed to be available in simulation results shown in Figs. 4-8. For the practical channel/beamformer estimation results shown in Figs. 9-11 the pilot gain is considered to be $10 \mathrm{~dB}$.

The actual achievable sum rate obtained from the WSR maximization objective versus the total overhead due to OTA signaling is shown in top of the Fig. 4. The second figure illustrates the convergence of the beamformer algorithm with respect to the number of beamformer iterations. In this particular example, we assume that a single precoder/decoder iteration consumes $1 \%\left(\frac{\tau_{0}}{T}=0.01\right)$ of the frame length. Note that, it is possible to have $\tau_{0}$ smaller than $0.1 \mathrm{~ms}$ with the proposed TDD frame structure [18]. Hence, we can obtain $\frac{\tau_{0}}{T}=0.01$ even with relatively short coherence time (if the coherence time is $10 \mathrm{~ms}$, we can have a TDD frame with $\mathrm{T}=10$ ms). We can observe that the scenario with $\beta=30, \zeta=0.05$ and $\theta=e$ has a better converging behaviour in comparison 

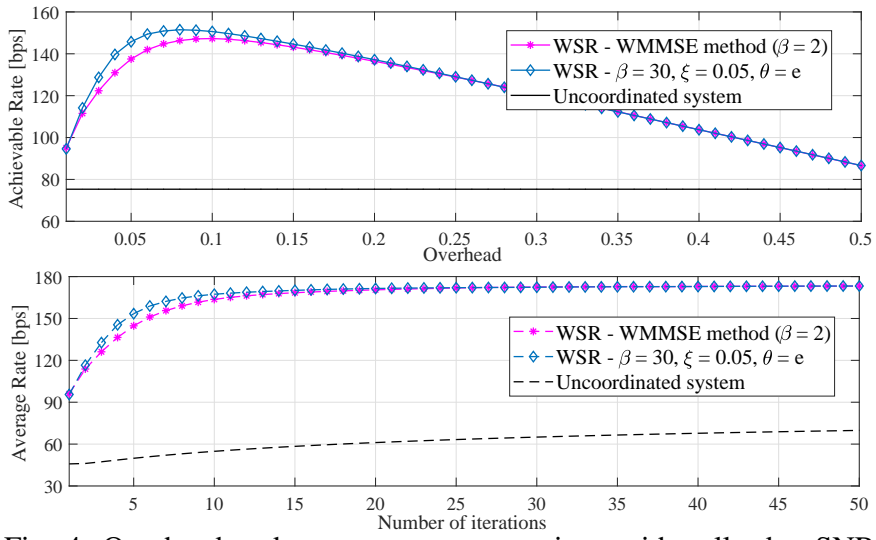

Fig. 4: Overhead and convergence comparison with cell edge SNR $=20 \mathrm{~dB}, K=152, K_{i}=8 \& M_{i}=8 \forall i \in \mathcal{B}, N_{k}=2 \forall k$.

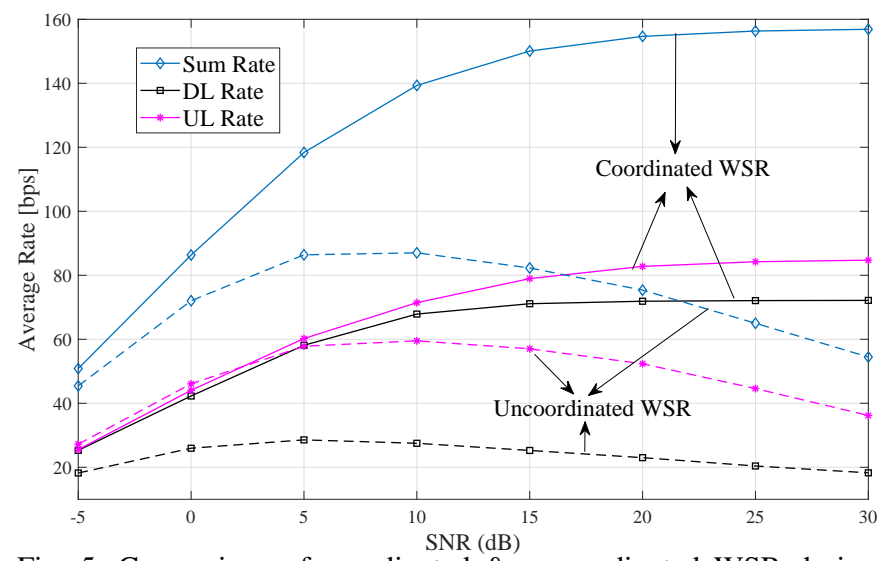

Fig. 5: Comparison of coordinated \& uncoordinated WSR designs with $K=152, K_{i}=8 \& M_{i}=8 \forall i \in \mathcal{B}, N_{k}=2 \forall k$.

to WMMSE [24] approach. Also, the coordinated system provides considerable gain as compared to the uncoordinated system. The uncoordinated beamformer design is introduced as a reference, where the beamformers are calculated locally without considering the inter-cell interference. It shows that the achievable rate peaks, when the overhead is between $0.05-0.10$, i.e., BIT $=5-10$ rounds. The achieved gain over the uncoordinated scheme is almost $100 \%$ due to the greatly improved interference coordination. For the rest of the figures, we will use parameters $\beta=30, \zeta=0.05, \theta=e$ and $\mathrm{BIT}=5$.

Fig. 5 illustrates the average rate of the dynamic TDD system versus the transmit SNR considering the DL, UL and UL/DL sum rates. Note that the results are shown after 5 precoder/decoder iterations. We can observe that the sum rate improves for both coordinated and uncoordinated systems with SNR up to $5 \mathrm{~dB}$. After that the uncoordinated system performance degrades due to the strong interference from the other-cell users. However, the proposed coordinated scheme performs well even at high SNR region. Due to user specific power constraints, the each UL user uses all the power for transmit beamforming. This leads to high UL-to-DL interference, which degrades the DL user SINR and rate. Thus, the UL users have somewhat higher rate at high SNR. Fig. 6,

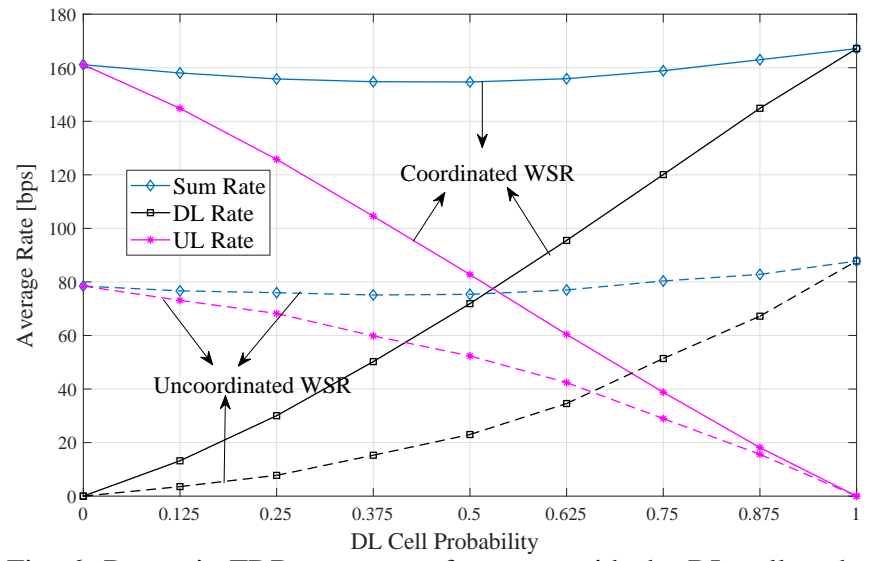

Fig. 6: Dynamic TDD system performance with the DL cell probability at cell edge $\mathrm{SNR}=20 \mathrm{~dB}, K=152, K_{i}=8 \& M_{i}=8 \forall i \in$ $\mathcal{B}, N_{k}=2 \forall k$.

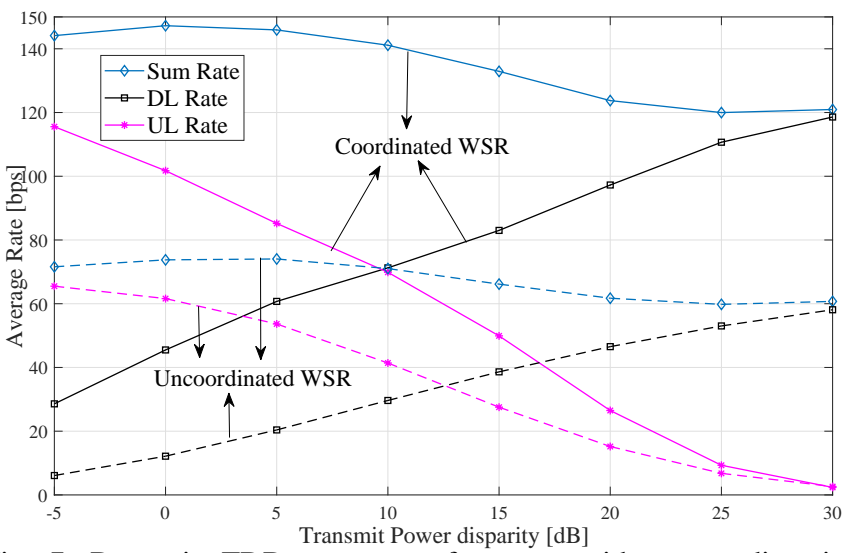

Fig. 7: Dynamic TDD system performance with power disparity between DL and UL transmissions at cell edge SNR $=20 \mathrm{~dB}$, $K_{i}=4 M_{i}=8 \forall i \in \mathcal{B}, N_{k}=2 \forall k$.

represents average rate performance of the dynamic TDD system with given DL cell probability. Despite difficult UL-DL interference scenarios, the sum rate is only slightly decreased (around $5-6 \%$ ) with 0.5 DL cell probability as compared to the DL or UL only cases.

The average rate of the dynamic TDD system against the power disparity between DL and UL transmissions is shown in Fig. 7. Power disparity is defined as $10 \log _{10}\left(P_{i}^{(\mathrm{dl})} / P_{k}^{(\mathrm{ul})}\right)$ $\mathrm{dB}$. We change the UL power while keeping DL power fixed to obtain different disparity levels. We can observe that the UL rate degrades and DL rate increases linearly with the power disparity. Also, UL and DL rates show similar performance, when the power disparity is $10 \mathrm{~dB}$. Similar to previous cases, the coordinated design show $100 \%$ gain in comparison to the uncoordinated case in all disparity levels.

In Fig. 8, we compare the proposed beamformer objectives with respect to total backlogged packets in the system queues for a given traffic arrival rate. As we study the system for time-correlated fading, we can use beamformers estimated in the previous time slot to initialize the beamformers for the current time slot (with memory). Thus, we can improve the beamformer convergence. Note that, this is only useful when our objectives are WQM or QWSR maximization. In 


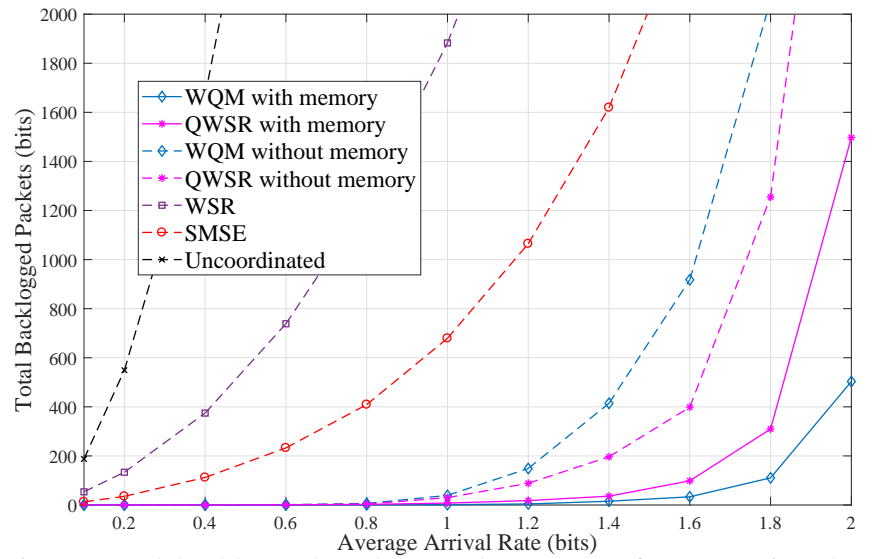

Fig. 8: Total backlogged packets at the system after 1000 timeslots for each optimization objective with cell edge SNR $=20 \mathrm{~dB}, K=$ $38, K_{i}=2 \& M_{i}=4 \forall i \in \mathcal{B}, N_{k}=2 \forall k$.

other objectives 'with memory' approach works only when every user has a large queue. Here, WQM provides better performance with memory compared to other methods, due to the improved convergence properties. However, QWSR provides good performance in both 'with memory' and 'without memory' approaches due to its fast rate of convergence. Interestingly, (W)SR has very poor performance, since it ignores the backlog status in the user queues altogether and tends to assign non-zero weights $\omega_{k, l}^{(a)}$ only to a subset of users with good channel (and interference) conditions. On the other hand, using SMSE criterion results in somewhat more fair resource allocation as $\omega_{k, l}^{(a)}=1 \forall k, l$. As a result, the traffic aware (WQM, QWSR) beamformer design can handle up to $2-3$ or 10 times larger traffic loads than traditional (W)SR and SMSE criteria or uncoordinated design, respectively.

In Fig. 9, the performance of the proposed bi-directional direct beamformer estimation strategies are illustrated with the SSE approach. In general, we do not assume any pilot coordination between cells. Therefore, the user specific training sequences in both forward and backward directions are nonorthogonal (overlapping). However, the pilots for same cell users can be still made orthogonal. Thus, in Fig. 9 each pilot sequence is interfered by up to 72 partially overlapping pilot sequences. For short sequence lengths, the received precoded pilot matrix is heavily contaminated by pilot interference. Especially, Strategy C does not perform well in these conditions due to significant mismatch between the received pilot matrices $\mathbf{R}_{k}^{(a)}$ and $\mathbf{R}_{k, 2}^{(a)}$ required to compute the beamformers in (40). Strategy B is less prone to errors in updating the weights $\omega_{k, l}^{(a)}$ as they are estimated only for intra-cell users. Therefore, it performs somewhat better than Strategy A with short sequence lengths. However, its performance saturates with less overlapping pilots as the user weights from other cell users are not available. The proposed strategies A and B start to perform reasonably well when the sequence length is longer than 24. In general, the DE approach provides significantly better performance than the SSE method. Moreover, due to the improved decontamination ability discussed in Section IV-E, it is able to provide the same sum rate as the SSE method with much shorter $(\leq 1 / 2)$ pilot sequence lengths.

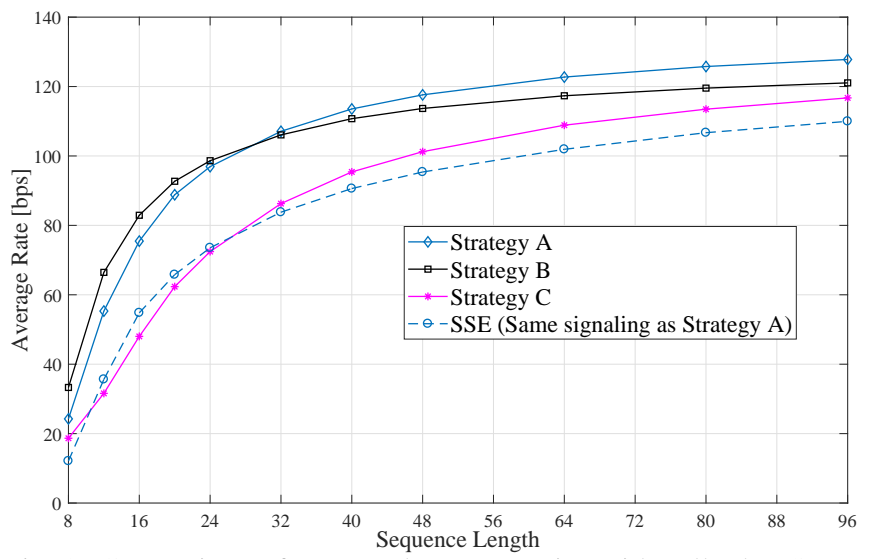

Fig. 9: Comparison of proposed DE strategies with cell edge SNR = $20 \mathrm{~dB}, N=19, K=76, K_{i}=4 \& M_{i}=8 \forall i \in \mathcal{B}, N_{k}=2 \forall k$.

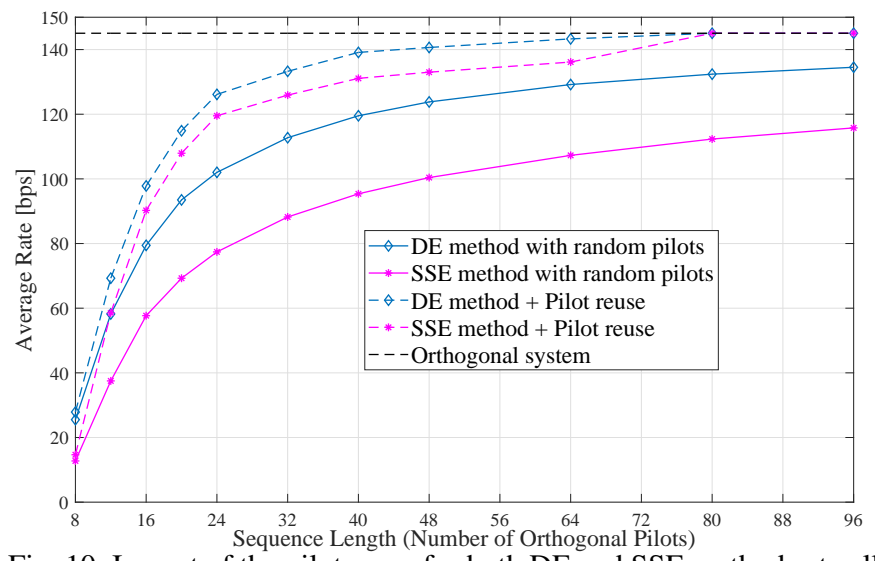

Fig. 10: Impact of the pilot reuse for both DE and SSE methods at cell edge $\mathrm{SNR}=20 \mathrm{~dB}, K=76, K_{i}=4 \& M_{i}=8 \forall i \in \mathcal{B}, N_{k}=2 \forall k$.

Fig. 10 illustrates the added value of centralized pilot allocation given in Algorithm 2 for both DE and SSE methods (both with Strategy A signaling). As expected, the centralized pilot allocation, requiring tight resource coordination (via backhaul) among adjacent cells, further improves the performance of both beamformer estimation schemes. Especially, the performance of the SSE method is greatly improved due to minimized pilot overlap. However, an uncoordinated random pilot allocation combined with the proposed direct beamformer estimation framework can provide most of these gains while requiring minimum or no coordination. Note that, in this example, the number of data streams per user is restricted to 1 . Therefore, every stream can be allocated with an orthogonal pilot for the bi-directional training when the pilot length is greater than 76. Finally, In Fig 11, the performance of the Dynamic TDD system by employing both Strategy A and pilot reuse is illustrated for different UE/BS densities $\left(K_{i}\right)$. There is a significant rate loss for the $K_{i}=8$ case, when the number of pilots is less than 20 . There, for number of pilots $8-20$, sum rate of the system is in the range of $10 \%-60 \%$ in comparison to the orthogonal system. However, note that this is due to the severe pilot contamination of the system, where same pilot will be shared with more than 8 users. For all the UE/BS densities, system performance is quite satisfactory when the pilots/user 


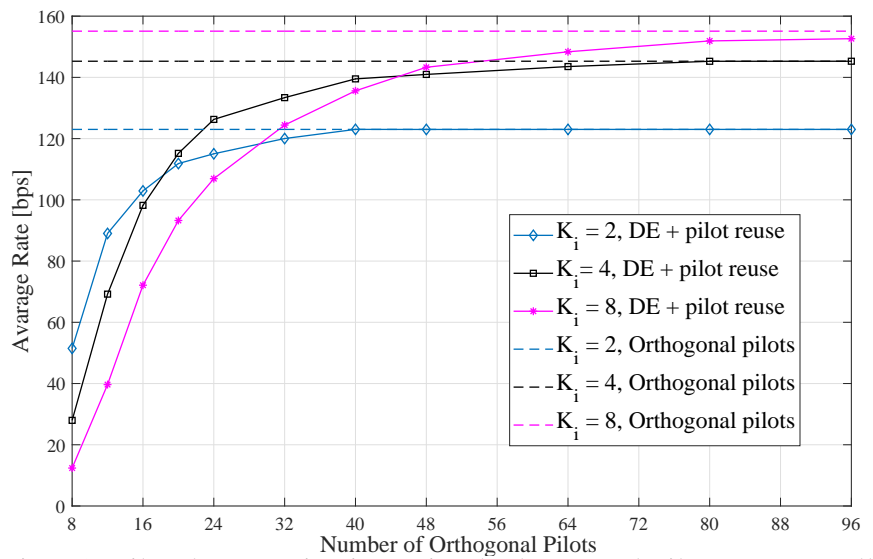

Fig. 11: Pilot decontamination using both $\mathrm{DE}$ and pilot reuse at cell edge $\mathrm{SNR}=20 \mathrm{~dB}, \quad M_{i}=8 \forall i \in \mathcal{B}, N_{k}=2 \forall k$

ratio is greater than $25 \%$.

\section{CONCLUSiOnS}

Multiantenna interference management for dynamic TDD systems was investigated with several network optimization objectives. Bi-directional OTA signaling and training framework was developed to iteratively optimize the transmit and receive beamformers both in UL and DL. Distributed fast converging beamformer design based on the WQM criterion was shown to be to the best approach for handling both dynamic traffic variation and difficult interference scenarios. Three DE strategies were proposed to alleviate the contamination due to non-orthogonal and overlapping pilot allocation. The Strategies A and B were shown to perform reasonably well even with relatively short pilot sequence lengths. Finally, a centralized pilot allocation scheme was introduced to further enhance the pilot decontamination. The numerical results demonstrate that the proposed training and estimation framework provides superior system performance over the uncoordinated scheme for different dynamic TDD network parameters and optimization objectives.

\section{REFERENCES}

[1] C. Systems. Cisco Visual Networking Index: Forecast and Methodology, 2016-2021. [Online Sep. 2017], https://www.cisco.com/c/en/us/ solutions/collateral/service-provider/visual-networking-index-vni/ complete-white-paper-c11-481360.pdf.

[2] J. A. Stankovic, "Research Directions for the Internet of Things," IEEE Internet Things J., vol. 1, no. 1, pp. 3-9, Mar. 2014.

[3] T. Nakamura, S. Nagata, A. Benjebbour, Y. Kishiyama, T. Hai, S. Xiaodong, Y. Ning, and L. Nan, "Trends in small cell enhancements in LTE advanced," IEEE Commun. Mag., vol. 51, no. 2, pp. 98-105, Feb. 2013.

[4] S. Chen and J. Zhao, "The requirements, challenges, and technologies for $5 \mathrm{G}$ of terrestrial mobile telecommunication," IEEE Commun. Mag., vol. 52, no. 5, pp. 36-43, May. 2014.

[5] J. Li, S. Farahvash, M. Kavehrad, and R. Valenzuela, "Dynamic TDD and fixed cellular networks," IEEE Commun. Lett., vol. 4, no. 7, pp. 218-220, Jul. 2000

[6] Z. Shen, A. Khoryaev, E. Eriksson, and X. Pan, "Dynamic uplinkdownlink configuration and interference management in TD-LTE," IEEE Commun. Mag., vol. 50, no. 11, pp. 51-59, Nov. 2012.

[7] B. Yu, S. Mukherjee, H. Ishii, and L. Yang, "Dynamic TDD support in the LTE-B enhanced Local Area architecture," in IEEE Globecom Workshops, 2012, pp. 585-591.
[8] 3GPP TSG RAN WG1, "Study on scenarios and requirements for next generation access technologies TR 38.913," 3rd Generation Partnership Project 3GPP, www.3gpp.org, Tech. Rep., 2017.

[9] 3GPP, "Technical report 38.802: Study on new radio access technology physical layer aspects v.2.0.0," 3rd Generation Partnership Project 3GPP, www.3gpp.org, Tech. Rep., Mar. 2017.

[10] E. Lahetkangas, K. Pajukoski, J. Vihriala, G. Berardinelli, M. Lauridsen, E. Tiirola, and P. Mogensen, "Achieving low latency and energy consumption by $5 \mathrm{G}$ TDD mode optimization," in IEEE Int. Conf. Commun. Workshops (ICC). IEEE, jun. 2014, pp. 1-6.

[11] P. Omiyi, H. Haas, and G. Auer, "Analysis of TDD cellular interference mitigation using busy-bursts," IEEE Trans. Wireless Commun., vol. 6, no. 7, pp. 2721-2731, Jul. 2007.

[12] Y. Choi, I. Sohn, and K. B. Lee, "A novel decentralized time slot allocation algorithm in dynamic TDD system," in IEEE Consumer Communications and Networking Conference (CCNC), vol. 2, 2006, pp. $1268-1272$.

[13] I. Sohn, K. B. Lee, and Y. Choi, "Comparison of decentralized time slot allocation strategies for asymmetric traffic in TDD systems," IEEE Trans. Wireless Commun., vol. 8, no. 6, pp. 2990-3003, Jun. 2009.

[14] H. Chung, M. Kim, N. Kim, and S. Yun, "Time slot allocation based on region and time partitioning for dynamic TDD-OFDM systems," in IEEE Vehi. Tech. Conf. (VTC), vol. 5, 2006, pp. 2459-2463.

[15] M. S. ElBamby, M. Bennis, W. Saad, and M. Latva-aho, "Dynamic Uplink-Downlink Optimization in TDD-based Small Cell Networks," in IEEE Int. Symposium on Wireless Communication Systems (ISWCS), 2014.

[16] K. Lee, Y. Park, M. Na, H. Wang, and D. Hong, "Aligned Reverse Frame Structure for Interference Mitigation in Dynamic TDD Systems," IEEE Trans. Wireless Commun., vol. 16, no. 10, pp. 6967-6978, Aug. 2017.

[17] P. Komulainen, A. Tölli, and M. Juntti, "Effective CSI signaling and decentralized beam coordination in TDD multi-cell MIMO systems," IEEE Trans. Signal Processing, vol. 61, no. 9, pp. 2204-2218, May. 2013.

[18] A. Tölli, H. Ghauch, J. Kaleva, P. Komulainen, M. Bengtsson, M. Skoglund, M. Honig, E. Lähetkangas, E. Tiirola, and K. Pajukoski, "Distributed Coordinated Transmission with Forward-Backward Training for 5G Radio Access," IEEE Commun. Mag. (major revision), Feb. 2018. [Online]. Available: https://arxiv.org/abs/1802.04309

[19] T. L. Marzetta, "Noncooperative cellular wireless with unlimited numbers of base station antennas," IEEE Trans. Wireless Commun., vol. 9, no. 11 , pp. $3590-3600$, Oct. 2010

[20] E. G. Larsson, O. Edfors, F. Tufvesson, and T. L. Marzetta, "Massive MIMO for next generation wireless systems," IEEE Commun. Mag., vol. 52, no. 2, pp. 186-195, Feb. 2014.

[21] M. Ding, D. L. Perez, A. V. Vasilakos, and W. Chen, "Dynamic TDD transmissions in homogeneous small cell networks," in IEEE Int. Conf. Commun. Workshops (ICC). IEEE, 2014, pp. 616-621.

[22] C. Yoon and D. H. Cho, "Energy efficient beamforming and power allocation in dynamic TDD based C-RAN system," IEEE Commun. Lett., vol. 19, no. 10, pp. 1806-1809, Aug. 2015.

[23] S. S. Christensen, R. Agarwal, E. Carvalho, and J. M. Cioffi, "Weighted sum-rate maximization using weighted MMSE for MIMO-BC beamforming design," IEEE Trans. Wireless Commun., vol. 7, no. 12, pp. 4792-4799, Dec. 2008

[24] Q. Shi, M. Razaviyayn, Z. Luo, and C. He, "An iteratively weighted MMSE approach to distributed sum-utility maximization for a MIMO interfering broadcast channel," IEEE Trans. Signal Processing, vol. 59, no. 9, pp. 4331-4340, Sept. 2011.

[25] D. A. Schmidt, C. Shi, R. A. Berry, M. L. Honig, and W. Utschick, "Comparison of distributed beamforming algorithms for MIMO interference networks," IEEE Trans. Signal Processing, vol. 61, no. 13, pp. 3476-3489, Jul. 2013.

[26] J. Kaleva, A. Tölli, and M. Juntti, "Decentralized sum rate maximization with QoS constraints for interfering broadcast channel via successive convex approximation," IEEE Trans. Signal Processing, vol. 64, no. 11, pp. 2788-2802, Jun. 2016.

[27] G. Venkatraman, A. Tölli, M. Juntti, and L. N. Tran, "Traffic aware resource allocation schemes for multi-cell MIMO-OFDM systems," IEEE Trans. Signal Processing, vol. 64, no. 11, pp. 2730-2745, Jun. 2016.

[28] R. A. Berry and E. M. Yeh, "Cross-layer wireless resource allocation," IEEE Signal Processing mag., vol. 21, no. 5, pp. 59-68, Aug. 2004.

[29] M. Chiang, S. H. Low, A. R. Calderbank, and J. C. Doyle, "Layering as optimization decomposition: A mathematical theory of network architectures," Proceedings of the IEEE, vol. 95, no. 1, pp. 255-312, Mar. 2007. 
[30] O. Tervo, L. N. Tran, and M. Juntti, "Optimal energy-efficient transmit beamforming for multi-user MISO downlink," IEEE Trans. Signal Processing, vol. 63, no. 20, pp. 5574-5588, Jul. 2015.

[31] M. C. Filippou, P. De Kerret, D. Gesbert, T. Ratnarajah, A. Pastore, and G. A. Ropokis, "Coordinated shared spectrum precoding with distributed CSIT," IEEE Trans. on Wireless Commun., vol. 15, no. 8, pp. 51825192, Apr. 2016.

[32] J. Jose, A. Ashikhmin, P. Whiting, and S. Vishwanath, "Channel estimation and linear precoding in multiuser multiple-antenna TDD systems," IEEE Trans. Vehi. Tech., vol. 60, no. 5, pp. 2102-2116, Apr. 2011.

[33] M. Biguesh and A. B. Gershman, "Training-based MIMO channel estimation: a study of estimator tradeoffs and optimal training signals," IEEE Trans. Signal Processing, vol. 54, no. 3, pp. 884-893, Feb. 2006.

[34] C. Shi, R. A. Berry, and M. L. Honig, "Bi-Directional Training for Adaptive Beamforming and Power Control in Interference Networks," IEEE Trans. Signal Processing, vol. 62, pp. 607-618, Feb. 2014.

[35] R. Brandt and M. Bengtsson, "Distributed CSI acquisition and coordinated precoding for TDD multicell MIMO systems," IEEE Trans. Vehi. Tech., vol. 65, no. 5, pp. 2890-2906, May. 2016.

[36] R. R. Müller, L. Cottatellucci, and M. Vehkaperä, "Blind pilot decontamination," IEEE J. Select Topics Signal Processing, vol. 8, no. 5, pp. 773-786, Mar. 2014.

[37] H. Yin, D. Gesbert, M. C. Filippou, and Y. Liu, "Decontaminating pilots in massive MIMO systems," in IEEE Int. Conf. Commun. (ICC). IEEE, 2013, pp. 3170-3175.

[38] H. Q. Ngo, T. L. Marzetta, and E. G. Larsson, "Analysis of the pilot contamination effect in very large multicell multiuser MIMO systems for physical channel models," in IEEE Int. Conf. Acoustics, Speech and Signal Processing (ICASSP), 2011, pp. 3464-3467.

[39] J. Jose, A. Ashikhmin, T. L. Marzetta, and S. Vishwanath, "Pilot contamination and precoding in multi-cell TDD systems," IEEE Trans. Wireless Commun., vol. 10, no. 8, pp. 2640-2651, Aug. 2011.

[40] E. Björnson, J. Hoydis, and L. Sanguinetti, "Pilot contamination is not a fundamental asymptotic limitation in massive MIMO," in IEEE Int. Conf. Commun. (ICC), May 2017, pp. 1-6.

[41] H. Xu, C. Pan, W. Xu, G. L. Stuber, J. Shi, and M. Chen, "Robust Beamforming with Pilot Reuse Scheduling in a Heterogeneous Cloud Radio Access Network," IEEE Trans. Vehi. Tech., Apr. 2018.

[42] P. Jayasinghe, A. Tölli, J. Kaleva, and M. Latva-aho, "Bi-directiona signaling for dynamic TDD with decentralized beamforming," in IEEE Int. Conf. Commun. Workshop (ICC), 2015, pp. 185-190.

[43] P. Jayasinghe, A. Tölli, and M. Latva-aho, "Bi-directional signaling strategies for dynamic TDD networks," in IEEE Int. Workshp Signal Processing Advances Wireless Commun. (SPAWC), 2015, pp. 540-544.

[44] P. Jayasinghe, A. Tölli, J. Kaleva, and M. Latva-aho, "Direct beamformer estimation for dynamic TDD networks with forward-backward training," in IEEE Int. Workshp Signal Processing Advances Wireless Commun. (SPAWC). IEEE, Jul. 2017, pp. 1-6.

[45] P. Jayasinghe, A. Tölli, J. Kaleva, G. Venkatraman, and M. Latva-aho, "Traffic aware pilot de-contamination for multi-cell MIMO systems," in European Conference on Networks and Communications (EuCNC). IEEE, Jun. 2017, pp. 1-6.

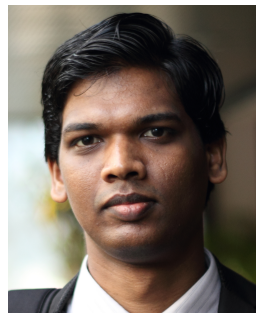

Praneeth Jayasinghe (S'13) received the B.Sc (Eng.) degree in electronics and telecommunications engineering from the University of Moratuwa, Sri Lanka in 2009 and M.Sc.(Tech.) degree in wireless communication engineering from University of Oulu, Oulu, Finland in 2014.

$\mathrm{He}$ is currently a doctoral student at the Department of Communication Engineering, University of Oulu, Oulu, Finland. His studies are mainly focused on resource allocation on flexible TDD based heterogeneous wireless networks.

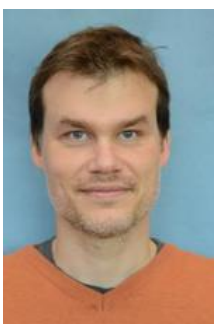

Antti Tlli (M'08, SM'14) received the Dr.Sc. (Tech.) degree in electrical engineering from the University of Oulu, Oulu, Finland, in 2008. Before joining the Centre for Wireless Communications (CWC) at the University of Oulu, he worked for 5 years with Nokia Networks as a Research Engineer and Project Manager both in Finland and Spain. In May 2014, he was granted a five year (2014 - 2019) Academy Research Fellow post by the Academy of Finland. Currently, he also holds an Associate Professor position with the University of Oulu. During the academic year 2015 - 2016, he visited at EURECOM, Sophia Antipolis, France, while from August 2018 till June 2019 he is visiting University of California - Santa Barbara, USA. He has authored more than 160 papers in peer-reviewed international journals and conferences and several patents all in the area of signal processing and wireless communications. His research interests include radio resource management and transceiver design for broadband wireless communications with a special emphasis on distributed interference management in heterogeneous wireless networks. He is currently serving as an Associate Editor for IEEE Transactions on Signal Processing.

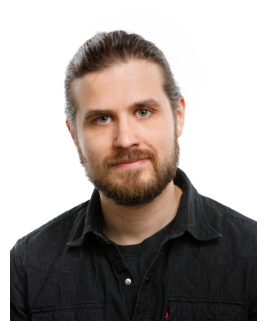

Jarkko Kaleva (S'11-M'18) received his Dr.Sc. (Tech.) degree in communications engineering from University of Oulu, Oulu, Finland in 2018 with distinction. In 2010, he joined Centre for Wireless Communications (CWC) at University of Oulu, Finland, where he is currently working as a postdoctoral researcher. His main research interests are in radio resource management for wireless communication and nonlinear programming.

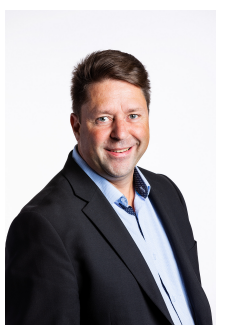

Matti Latva-aho (S'96-M'98-SM'06) received the M.Sc., Lic.Tech. and Dr. Tech (Hons.) degrees in Electrical Engineering from the University of Oulu, Finland in 1992, 1996 and 1998, respectively. From 1992 to 1993 , he was a Research Engineer at Nokia Mobile Phones, Oulu, Finland after which he joined Centre for Wireless Communications (CWC) at the University of Oulu. Prof. Latva-aho was Director of CWC during the years $1998-2006$ and Head of Department for Communication Engineering until August 2014. Currently he is Professor of Digital Transmission Techniques at the University of Oulu. He serves as Academy of Finland Professor in 2017 - 2022. His research interests are related to mobile communication systems and currently his group focuses on $5 \mathrm{G}$ and beyond systems research. Prof. Latva-aho is Director for 6G Wireless Flagship in Finland (www.6genesis.org) in 2018 - 2026. Prof. Latva-aho has published $350+$ conference or journal papers in the field of wireless communications. He received Nokia Foundation Award in 2015 for his achievements in mobile communications research. 